\title{
KARCHER MEANS AND KARCHER EQUATIONS OF POSITIVE DEFINITE OPERATORS
}

\author{
JIMMIE LAWSON AND YONGDO LIM
}

\begin{abstract}
The Karcher or least-squares mean has recently become an important tool for the averaging and studying of positive definite matrices. In this paper we show that this mean extends, in its general weighted form, to the infinite-dimensional setting of positive operators on a Hilbert space and retains most of its attractive properties. The primary extension is via its characterization as the unique solution of the corresponding Karcher equation. We also introduce power means $P_{t}$ in the infinite-dimensional setting and show that the Karcher mean is the strong limit of the monotonically decreasing family of power means as $t \rightarrow 0^{+}$. We show that each of these characterizations provide important insights about the Karcher mean.
\end{abstract}

\section{INTRODUCTION}

Positive definite matrices have become fundamental computational objects in many areas of engineering, statistics, quantum information, and applied mathematics. They appear as "data points" in a diverse variety of settings: covariance matrices in statistics, elements of the search space in convex and semidefinite programming, kernels in machine learning, density matrices in quantum information, and diffusion tensors in medical imaging, to cite only a few. A variety of computational algorithms have arisen for approximations, interpolation, filtering, estimation, and averaging.

The process of averaging typically involves taking some type of matrix mean for some finite number of positive matrices of fixed dimension. Since the pioneering paper of Kubo and Ando [1], an extensive theory of two-variable means has sprung up for positive matrices and operators, but the $n$-variable case for $n>2$ has remained problematic. Once one realizes, however, that the matrix geometric mean $g_{2}(A, B)=A \# B:=A^{1 / 2}\left(A^{-1 / 2} B A^{-1 / 2}\right)^{1 / 2} A^{1 / 2}$ is the metric midpoint of $A$ and $B$ for the trace metric on the set $\mathbb{P}$ of positive definite matrices of some fixed dimension (see, e.g., 4, 13 ), it is natural to use an averaging technique over this metric to extend this mean to a larger number of variables. First M. Moakher [18] and then Bhatia and Holbrook [5] suggested extending the geometric mean to $n$-points by taking the mean to be the unique minimizer of the sum of the squares

Received by the editors May 18, 2012 and, in revised form, November 22, 2012.

2010 Mathematics Subject Classification. Primary 47B65; Secondary 47L07, 15A48.

Key words and phrases. Positive operator, operator mean, Karcher equation, Karcher mean, power mean.

The work of the second author was supported by the National Research Foundation of Korea (NRF) grant funded by the Korean government (MEST) (No. 2012-005191). 
of the distances:

$$
g_{n}\left(A_{1}, \ldots, A_{n}\right)=\underset{X>0}{\arg \min } \sum_{i=1}^{n} \delta^{2}\left(X, A_{i}\right),
$$

where $\delta\left(X, A_{i}\right)=\left\|\log X^{-1 / 2} A_{i} X^{-1 / 2}\right\|$. This idea had been anticipated by Élie Cartan (see, for example, section 6.1 .5 of 2 ), who showed among other things such a unique minimizer exists if the points all lie in a convex ball in a Riemannian manifold, which is enough to deduce the existence of the least-squares mean globally for $\mathbb{P}$. A more detailed study of Riemannian centers of mass in the setting of Riemannian manifolds was carried out by H. Karcher [10], whose ideas are important to the present work.

Another approach to generalizing the geometric mean to $n$-variables, independent of metric notions, was suggested by Ando, Li, and Mathias [1] via a "symmetrization procedure" and induction. The Ando-Li-Mathias paper was also important for listing, and deriving for their mean, ten desirable properties for extended geometric means. Moaker and Bhatia and Holbrook were able to establish a number of these important properties for the least-squares mean, but the important question of the monotonicity of this mean, conjectured by Bhatia and Holbrook [5], was left open. However, the authors were recently able to show [16 that all the properties, in particular the monotonicity, were satisfied in the more general setting of weighted means for any weight $\omega=\left(w_{1}, \ldots w_{n}\right)$ of non-negative entries summing to 1:

(P1) (Consistency with scalars) $g_{n}(\omega ; \mathbb{A})=A_{1}^{w_{1}} \cdots A_{n}^{w_{n}}$ if the $A_{i}$ 's commute;

(P2) (Joint homogeneity) $g_{n}\left(\omega ; a_{1} A_{1}, \ldots, a_{n} A_{n}\right)=a_{1}^{w_{1}} \cdots a_{n}^{w_{n}} g_{n}(\omega ; \mathbb{A})$;

(P3) (Permutation invariance)

$$
g_{n}\left(\omega_{\sigma} ; \mathbb{A}_{\sigma}\right)=g(\omega ; \mathbb{A}), \text { where } \omega_{\sigma}=\left(w_{\sigma(1)}, \ldots, w_{\sigma(n)}\right) ;
$$

(P4) (Monotonicity) if $B_{i} \leq A_{i}$ for all $1 \leq i \leq n$, then $g_{n}(\omega ; \mathbb{B}) \leq g_{n}(\omega ; \mathbb{A})$;

(P5) (Continuity) the map $g_{n}(\omega ; \cdot)$ is continuous;

(P6) (Congruence invariance) $g_{n}\left(\omega ; M^{*} \mathbb{A} M\right)=M^{*} g_{n}(\omega ; \mathbb{A}) M$ for any invertible $M$;

(P7) (Joint concavity) $g_{n}(\omega ; \lambda \mathbb{A}+(1-\lambda) \mathbb{B}) \geq \lambda g_{n}(\omega ; \mathbb{A})+(1-\lambda) g_{n}(\omega ; \mathbb{B})$ for $0 \leq \lambda \leq 1$

(P8) (Self-duality) $g_{n}\left(\omega ; A_{1}^{-1}, \ldots, A_{n}^{-1}\right)^{-1}=g_{n}\left(\omega ; A_{1}, \ldots, A_{n}\right)$;

(P9) (Determinental identity) $\operatorname{Det} g_{n}(\omega ; \mathbb{A})=\prod_{i=1}^{n}\left(\operatorname{Det} A_{i}\right)^{w_{i}}$; and

(P10) (AGH weighted mean inequalities)

$$
\left(\sum_{i=1}^{n} w_{i} A_{i}^{-1}\right)^{-1} \leq g_{n}(\omega ; \mathbb{A}) \leq \sum_{i=1}^{n} w_{i} A_{i} .
$$

A key ingredient in the derivation of many of these properties, the monotonicity in particular, is the fact that the trace metric on the manifold of positive definite matrices gives them the structure of a Cartan-Hadamard Riemannian manifold, in particular a manifold of non-positive curvature. This implies that equipped with the Riemannian distance metric the manifold is a metric space of non-positive curvature, an NPC-space for short, also called a $\mathrm{CAT}_{0}$-space, a widely studied class of metric spaces with a rich structure (see e.g., 6], 21, [12, Chapter 11]).

Since in the quantum as well as other settings, one is interested in the more general case of positive bounded linear operators on an infinite-dimensional Hilbert space, one would like to have suitable and effective averaging procedures for this 
context also. However, the significant theory that has developed for the multivariable least-squares mean does not readily carry over to the setting of positive operators on a Hilbert space, since one has no such Riemannian structure nor NPC-metric available. Nevertheless a natural question arises as to whether other (non-metric) characterizations of the least-squares mean generalize to the Hilbert space setting, a generalization that continues to satisfy the preceding properties $(\mathrm{P} 1)-(\mathrm{P} 8),(\mathrm{P} 10)$. In this paper we suggest and shall focus on an alternative characterization of the weighted least-squares mean as the unique solution of the Karcher equation

$$
\sum_{i=1}^{n} w_{i} \log \left(X^{-1 / 2} A_{i} X^{-1 / 2}\right)=0,
$$

show that this equation also has a unique positive solution in the infinite-dimensional setting, and thus refer to our generalization as the Karcher mean. (In the Riemannian setting the Karcher equation is the condition for the vanishing of the gradient of the least-squares distance function.)

In Section 2 we recall the Thompson metric and list properties of it that will be important for our development. Section 3 on power means introduces an important tool for later developments, but the fact that well-behaved power means exist for the Hilbert operator setting is of independent interest. Lim and Pálfia [17] have recently shown in the finite-dimensional setting that the Karcher or least-squares mean is the limit as $t \rightarrow 0^{+}$of the power means $P_{t}$. We show additionally that they are monotonically decreasing, which allows us to deduce the existence of their strong limit. In Section 4 we see that this power mean limit also exists in the Hilbert space setting and use this fact for our initial provisional definition of the Karcher mean. We show in Section 5 that the Karcher mean defined in this way does indeed satisfy the Karcher equation, and in Section 6 we establish that it is the unique solution and present a list of the fundamental properties of the Karcher mean.

Although for convenience we carry out our work in $\mathcal{B}(H)$, the $C^{*}$-algebra of all bounded linear operators on a Hilbert space $H$, our constructions only require that we be working in a monotone complete subalgebra of $\mathcal{B}(H)$, as we point out in the last section.

\section{The Thompson Metric}

For a Hilbert space $H$ let $\mathcal{B}(H)$ be the Banach space of bounded linear operators on $H$ equipped with the operator norm, $\mathcal{S}(H)$ the closed subspace of bounded self-adjoint linear operators, and let $\mathbb{P}=\mathbb{P}(H) \subseteq \mathcal{S}(H)$ be the open convex cone of positive definite operators. The Banach Lie group GL $(H)$ of bounded invertible linear operators (with operation composition) acts on $\mathbb{P}$ via congruence transformations: $\Gamma_{C}(X)=C X C^{*}$. For $X, Y \in \mathcal{S}(H)$, we write $X \leq Y$ if $Y-X$ is positive semidefinite, and $X<Y$ if $Y-X$ is positive definite. Note that $X \leq Y$ if and only if $\langle x, X x\rangle \leq\langle x, Y x\rangle$ for all $x \in H$.

For $A, B \in \mathbb{P}$ and $t \in \mathbb{R}$, the $t$-weighted geometric mean of $A$ and $B$ is defined by

$$
A \#{ }_{t} B=A^{1 / 2}\left(A^{-1 / 2} B A^{-1 / 2}\right)^{t} A^{1 / 2} .
$$

The following properties for the weighted geometric mean are well known [7, 11, 14]. 
Lemma 2.1. Let $A, B, C, D \in \mathbb{P}$ and let $t \in \mathbb{R}$. Then

(i) $A \#_{t} B=A^{1-t} B^{t}$ for $A B=B A$;

(ii) $(a A) \#_{t}(b B)=a^{1-t} b^{t}\left(A \#_{t} B\right)$ for $a, b>0$;

(iii) (Loewner-Heinz inequality) $A \#_{t} B \leq C \#_{t} D$ for $A \leq C, B \leq D$ and $t \in$ $[0,1]$

(iv) $M\left(A \#_{t} B\right) M^{*}=\left(M A M^{*}\right) \#_{t}\left(M B M^{*}\right)$ for $M \in \mathrm{GL}(H)$;

(v) $A \#_{t} B=B \#_{1-t} A,\left(A \#_{t} B\right)^{-1}=A^{-1} \#_{t} B^{-1}$;

(vi) $(\lambda A+(1-\lambda) B) \#_{t}(\lambda C+(1-\lambda) D) \geq \lambda\left(A \#_{t} C\right)+(1-\lambda)\left(B \#_{t} D\right)$ for $\lambda, t \in[0,1]$

(vii) $\left((1-t) A^{-1}+t B^{-1}\right)^{-1} \leq A \#{ }_{t} B \leq(1-t) A+t B$ for $t \in[0,1]$;

(viii) $\left(A \#_{t} B\right) \#_{s}\left(A \#_{u} B\right)=A \#(1-s) t+s u$ for any $s, t, u \in \mathbb{R}$. As a special case, $A \#_{s}\left(A \#_{t} B\right)=A \#_{s t} B$.

The Thompson metric on $\mathbb{P}$ is defined by $d(A, B)=\left\|\log \left(A^{-1 / 2} B A^{-1 / 2}\right)\right\|$, where $\|X\|$ denotes the operator norm of $X$. It is known that $d$ is a complete metric on $\mathbb{P}$ and that

$$
d(A, B)=\max \{\log M(B / A), \log M(A / B)\},
$$

where $M(B / A)=\inf \{\alpha>0: B \leq \alpha A\}$; see [7,20,22. We note that the Thompson metric (in the second form) exists on all normal cones of real Banach spaces. For instance,

$$
d\left(\left(s_{1}, \ldots, s_{n}\right),\left(t_{1}, \ldots, t_{n}\right)\right)=\max _{1 \leq i \leq n}\left|\log \frac{s_{i}}{t_{i}}\right|
$$

on $\mathbb{R}_{+}^{n}$, where $\mathbb{R}_{+}=(0, \infty)$.

Lemma 2.2 (3, 7, 14). Basic properties of the Thompson metric on $\mathbb{P}$ include

(i) $d(A, B)=d(r A, r B)=d\left(A^{-1}, B^{-1}\right)=d\left(M A M^{*}, M B M^{*}\right)$ for any $r>0$ and $M \in \mathrm{GL}(H)$;

(ii) $d(A \# B, A)=d(A \# B, B)=\frac{1}{2} d(A, B)$;

(iii) $d\left(A \#_{t} B, C \#_{t} D\right) \leq(1-t) d(A, C)+t d(B, D), \quad t \in[0,1]$;

(iv) $d\left(A \#_{t} B, A \#_{s} B\right)=|s-t| d(A, B), s, t \in[0,1]$.

By the triangular inequality,

$$
d\left(A \#_{t} B, C \#_{s} D\right) \leq(1-t) d(A, C)+t d(B, D)+|t-s| d(C, D)
$$

for all $s, t \in[0,1]$. Indeed,

$$
\begin{aligned}
d\left(A \#_{t} B, C \#_{s} D\right) & \leq d\left(A \#_{t} B, C \#_{t} D\right)+d\left(C \#_{t} D, C \#_{s} D\right) \\
\leq & (1-t) d(A, C)+t d(B, D)+|t-s| d(C, D) .
\end{aligned}
$$

The topology induced by the Thompson metric on $\mathbb{P}$ agrees with the relative Banach space topology [22]. It will be useful to have available some basic relationships between the operator norm and the Thompson metric. For this purpose we introduce the numerical radius of $A \in \mathcal{B}(H)$ :

$$
w(A):=\sup \{|\langle x, A x\rangle|: x \in H,\|x\|=1\} .
$$

Lemma 2.3. (i) For $A \in \mathcal{B}(H)$, $(1 / 2)\|A\| \leq w(A) \leq\|A\|$; the equality $w(A)=$ $\|A\|$ holds for $A \geq 0$ in $\mathcal{S}(H)$.

(ii) For $A, B \in \mathcal{S}(H), 0 \leq A$, and $\lambda, \mu \in \mathbb{R}, \lambda A \leq B \leq \mu A$ implies $w(B) \leq$ $\max \{|\lambda|,|\mu|\} w(A)$, and so $\|B\| \leq 2 \max \{|\lambda|,|\mu|\}\|A\|$. If $0 \leq B \leq A$, then $\|B\| \leq\|A\|$. 
Proof. (i) The inequality is a standard one; see, for example, [8]. For $B \in \mathcal{S}(H)$, we have for $\|x\|=1$ : $\langle B x, B x\rangle=\left\langle x, B^{2} x\right\rangle \leq w\left(B^{2}\right) \leq\left\|B^{2}\right\|=\|B\|^{2}$, the last equality coming from the fact $\mathcal{B}(H)$ is a $C^{*}$-algebra. Taking the supremum over all $\|x\|=1$ yields $\|B\|^{2} \leq w\left(B^{2}\right) \leq\left\|B^{2}\right\|=\|B\|^{2}$, so $w\left(B^{2}\right)=\left\|B^{2}\right\|$. Since any $A \geq 0$ has a (unique) square root $B \geq 0$, the second assertion follows.

(ii) For each $x \in H, \lambda\langle x, A x\rangle=\langle x, \lambda A x\rangle \leq\langle x, B x\rangle \leq\langle x, \mu A x\rangle=\mu\langle x, A x\rangle$, so $|\langle x, B x\rangle| \leq \max \{|\lambda|,|\mu|\} \mid\langle x, A x\rangle$. Taking sups over $\|x\|=1$ yields $w(B) \leq$ $\max \{|\lambda|,|\mu|\} w(A)$. The second assertion now follows from part (i). Taking $\lambda=0$, $\mu=1$ and using $w(C)=\|C\|$ for $C \geq 0$ from (i) yields the last inequality.

The following non-expansive property of addition for the Thompson metric will be useful for our purpose.

Lemma 2.4. [15, Lemma 10.1(iv)] Let $A_{i}, B_{i} \in \mathbb{P}, i=1,2, \ldots, n$. Then

$$
d\left(\sum_{i=1}^{n} A_{i}, \sum_{i=1}^{n} B_{i}\right) \leq \max _{1 \leq i \leq n}\left\{d\left(A_{i}, B_{i}\right)\right\} .
$$

Proof. For $n=2$, suppose that $d\left(A_{1}, B_{1}\right) \leq d\left(A_{2}, B_{2}\right)=\log r$. Then $A_{2} \leq r B_{2}$, $B_{2} \leq r A_{2}, A_{1} \leq r B_{1}, B_{1} \leq r A_{1}$, and thus $A_{1}+A_{2} \leq r B_{1}+r B_{2}=r\left(B_{1}+B_{2}\right), B_{1}+$ $B_{2} \leq r A_{1}+r A_{2}=r\left(A_{1}+A_{2}\right)$. Hence $d\left(A_{1}+A_{2}, B_{1}+B_{2}\right) \leq \log r=d\left(A_{2}, B_{2}\right)$. The general case easily follows by induction.

We denote by $\Delta_{n}$ the simplex of positive probability vectors in $\mathbb{R}^{n}$, the convex hull of the set of unit coordinate vectors. We equip $\Delta_{n}$ with the relative Thompson metric given by equation (2.2) (which induces the relative Euclidean topology). A map $G: \Delta_{n} \times \mathbb{P}^{n} \rightarrow \mathbb{P}$ is said to be monotonic if for any $\omega \in \Delta_{n}$,

$$
G\left(\omega ; A_{1}, \ldots, A_{n}\right) \leq G\left(\omega ; B_{1}, \ldots, B_{n}\right)
$$

whenever $A_{i} \leq B_{i}$ and $A_{i}, B_{i} \in \mathbb{P}$ for $i=1, \ldots, n$. A map $G: \Delta_{n} \times \mathbb{P}^{n} \rightarrow \mathbb{P}$ is said to be jointly homogeneous if $G\left(\omega ; a_{1} A_{1}, \ldots, a_{n} A_{n}\right)=a_{1}^{w_{1}} \cdots a_{n}^{w_{n}} G\left(\omega ; A_{1}, \ldots, A_{n}\right)$ for all $\omega=\left(w_{1}, \ldots, w_{n}\right) \in \Delta_{n}$ and $A_{i} \in \mathbb{P}, a_{i}>0, i=1, \ldots, n$.

Proposition 2.5. Let $G: \Delta_{n} \times \mathbb{P}^{n} \rightarrow \mathbb{P}$ be jointly homogeneous and monotonic. Then the following contractive property for the Thompson metric is satisfied:

$$
d(G(\omega ; \mathbb{A}), G(\omega ; \mathbb{B})) \leq \sum_{i=1}^{n} w_{i} d\left(A_{i}, B_{i}\right)
$$

for all $\omega=\left(w_{1}, \ldots, w_{n}\right) \in \Delta_{n}$ and $\mathbb{A}=\left(A_{1}, \ldots, A_{n}\right), \mathbb{B}=\left(B_{1}, \ldots, B_{n}\right) \in \mathbb{P}^{n}$.

Proof. By definition of the Thompson metric, $A_{i} \leq e^{d\left(A_{i}, B_{i}\right)} B_{i}$ and $B_{i} \leq e^{d\left(A_{i}, B_{i}\right)} A_{i}$ for all $i=1, \ldots, n$. By joint homogeneity and monotonicity of $G$,

$$
\begin{aligned}
G\left(\omega ; A_{1}, \ldots, A_{n}\right) & \leq G\left(\omega ; e^{d\left(A_{1}, B_{1}\right)} B_{1}, \ldots, e^{d\left(A_{n}, B_{n}\right)} B_{n}\right) \\
& =e^{\sum_{i=1}^{n} w_{i} d\left(A_{i}, B_{i}\right)} G\left(\omega ; B_{1}, \ldots, B_{n}\right)
\end{aligned}
$$

and similarly $G\left(\omega ; B_{1}, \ldots, B_{n}\right) \leq e^{\sum_{i=1}^{n} w_{i} d\left(A_{i}, B_{i}\right)} G\left(\omega ; A_{1}, \ldots, A_{n}\right)$. This implies that $d(G(\omega ; \mathbb{A}), G(\omega ; \mathbb{B})) \leq \sum_{i=1}^{n} w_{i} d\left(A_{i}, B_{i}\right)$. 


\section{Power meAns}

Power means for positive definite matrices have recently been introduced by Lim and Pálfia [17. Their notion and most of their results readily extend to the setting of positive operators on a Hilbert space, as we point out in this section.

Theorem 3.1. Let $A_{1}, \ldots, A_{n} \in \mathbb{P}$ and let $\omega=\left(w_{1}, \ldots, w_{n}\right) \in \Delta_{n}$. Then for each $t \in(0,1]$, the following equation has a unique positive definite solution:

$$
X=\sum_{i=1}^{n} w_{i}\left(X \#_{t} A_{i}\right) .
$$

Proof. We show that the map $f: \mathbb{P} \rightarrow \mathbb{P}$ defined by $f(X)=\sum_{i=1}^{n} w_{i}\left(X \#_{t} A_{i}\right)$ is a strict contraction with respect to the Thompson metric. Let $X, Y>0$. By Lemma 2.2(i),(iii) and Lemma 2.4.

$$
\begin{aligned}
d(f(X), f(Y)) & \leq \max _{1 \leq i \leq n}\left\{d\left(w_{i}\left(X \#_{t} A_{i}\right), w_{i}\left(Y \#_{t} A_{i}\right)\right)\right\} \\
& \leq \max _{1 \leq i \leq n}\left\{d\left(X \#_{t} A_{i}, Y \#_{t} A_{i}\right)\right\} \\
& \leq \max _{1 \leq i \leq n}\{(1-t) d(X, Y)\}=(1-t) d(X, Y) .
\end{aligned}
$$

Since $1-t<1, f$ is a strict contraction, hence has a unique fixed point.

Definition 3.2 (Power means). Let $\mathbb{A}=\left(A_{1}, \ldots, A_{n}\right) \in \mathbb{P}^{n}$ and $\omega \in \Delta_{n}$. For $t \in(0,1]$, we denote by $P_{t}(\omega ; \mathbb{A})$ the unique solution of

$$
X=\sum_{i=1}^{n} w_{i}\left(X \#_{t} A_{i}\right) .
$$

For $t \in[-1,0)$, we define $P_{t}(\omega ; \mathbb{A})=P_{-t}\left(\omega ; \mathbb{A}^{-1}\right)^{-1}$, where $\mathbb{A}^{-1}=\left(A_{1}^{-1}, \ldots, A_{n}^{-1}\right)$. We call $P_{t}(\omega ; \mathbb{A})$ the $\omega$-weighted power mean of order $t$ of $A_{1}, \ldots, A_{n}$.

Remark 3.3. We note that $P_{1}(\omega ; \mathbb{A})=\mathcal{A}(\omega ; \mathbb{A}):=\sum_{i=1}^{n} w_{i} A_{i}$ and $P_{-1}(\omega ; \mathbb{A})=$ $\mathcal{H}(\omega ; \mathbb{A}):=\left(\sum_{i=1}^{n} w_{i} A_{i}^{-1}\right)^{-1}$, the $\omega$-weighted arithmetic and harmonic means of $A_{1}, \ldots, A_{n}$, respectively. For $t \in[-1,0), P_{t}(\omega ; \mathbb{A})$ is the unique positive definite solution of

$$
X=\left[\sum_{i=1}^{n} w_{i}\left(X \#_{-t} A_{i}\right)^{-1}\right]^{-1} .
$$

Indeed, $X^{-1}=\sum_{i=1}^{n} w_{i}\left(X^{-1} \#_{-t} A_{i}^{-1}\right)$ if and only if $X^{-1}=P_{-t}\left(\omega ; \mathbb{A}^{-1}\right)$.

Remark 3.4. Let $t \in(0,1]$. Let $f: \mathbb{P} \rightarrow \mathbb{P}$ defined by $f(X)=\sum_{i=1}^{n} w_{i}\left(X \#_{t} A_{i}\right)$. Then by the Loewner-Heinz inequality, $f$ is monotone: $X \leq Y$ implies that $f(X) \leq$ $f(Y)$. By Theorem $3.1 f$ is a strict contraction for the Thompson metric with the least contraction coefficient less than or equal to $1-t$. By the Banach fixed point theorem

$$
\lim _{k \rightarrow \infty} f^{k}(X)=P_{t}(\omega ; \mathbb{A}), \quad X \in \mathbb{P} .
$$

Similarly, the map $g(X)=\left[\sum_{i=1}^{n} w_{i}\left(X \#_{t} A_{i}\right)^{-1}\right]^{-1}$ is a strict contraction for the Thompson metric and $\lim _{k \rightarrow \infty} g^{k}(X)=P_{-t}(\omega ; \mathbb{A})$, for any $X \in \mathbb{P}$. 
Proposition 3.5. Let $\omega, \mu \in \Delta_{n}$ and let $\mathbb{A}, \mathbb{B} \in \mathbb{P}^{n}$. Then for $t, s \in(0,1]$,

$$
d\left(P_{t}(\omega ; \mathbb{A}), P_{s}(\mu ; \mathbb{B})\right) \leq \frac{|s-t|}{\max \{s, t\}} \Delta(\mathbb{A})+\frac{1}{\max \{s, t\}} d(\omega, \mu)+\max _{1 \leq i \leq n} d\left(A_{i}, B_{i}\right),
$$

where $\Delta(\mathbb{A})=\max _{1 \leq i, j \leq n}\left\{d\left(A_{i}, A_{j}\right)\right\}$ denotes the d-diameter of $\mathbb{A}=\left(A_{1}, \ldots, A_{n}\right)$. In particular, the map $P:(0,1] \times \Delta_{n} \times \mathbb{P}^{n} \rightarrow \mathbb{P}$ defined by $P(t, \omega, \mathbb{A})=P_{t}(\omega ; \mathbb{A})$ is continuous with respect to the Thompson metric on $\mathbb{P}$.

Proof. Let $\omega=\left(w_{1}, \ldots, w_{n}\right), \mu=\left(u_{1}, \ldots, u_{n}\right) \in \Delta_{n}$ and $\mathbb{A}=\left(A_{1}, \ldots, A_{n}\right), \mathbb{B}=$ $\left(B_{1}, \ldots, B_{n}\right) \in \mathbb{P}^{n}$.

Step 1. $d\left(P_{t}(\omega ; \mathbb{A}), A_{j}\right) \leq \Delta(\mathbb{A})$ for all $j=1, \ldots, n$.

Let $X=P_{t}(\omega ; \mathbb{A})$. By Lemmas 2.2 and 2.4 ,

$$
\begin{aligned}
d\left(X, A_{j}\right) & =d\left(\sum_{i=1}^{n} w_{i}\left(X \#_{t} A_{i}\right), A_{j}\right)=d\left(\sum_{i=1}^{n} w_{i}\left(X \#_{t} A_{i}\right), \sum_{i=1}^{n} w_{i} A_{j}\right) \\
& \leq \max _{1 \leq i \leq n} d\left(X \#_{t} A_{i}, A_{j} \#_{t} A_{j}\right) \leq \max _{1 \leq i \leq n}\left[(1-t) d\left(X, A_{j}\right)+t d\left(A_{i}, A_{j}\right)\right] \\
& \leq(1-t) d\left(X, A_{j}\right)+t \Delta(\mathbb{A})
\end{aligned}
$$

and thus $d\left(X, A_{j}\right) \leq \Delta(\mathbb{A})$ for all $j=1, \ldots, n$.

Step 2. $d\left(P_{t}(\omega ; \mathbb{A}), P_{s}(\omega ; \mathbb{A})\right) \leq \frac{s-t}{s} \Delta(\mathbb{A})$ for $0<t \leq s \leq 1$.

Let $X=P_{t}(\omega ; \mathbb{A})$ and $Y=P_{s}(\omega ; \mathbb{A})$. By Lemma 2.4 and Step 1 ,

$$
\begin{aligned}
d(Y, X) & =d\left(\sum_{i=1}^{n} w_{i}\left(Y \#_{s} A_{i}\right), \sum_{i=1}^{n} w_{i}\left(X \#_{t} A_{i}\right)\right) \leq \max _{1 \leq i \leq n} d\left(Y \#_{s} A_{i}, X \#_{t} A_{i}\right) \\
& \stackrel{2.3}{\leq} \max _{1 \leq i \leq n}\left[(1-s) d(Y, X)+|s-t| d\left(X, A_{i}\right)\right] \\
& \leq(1-s) d(X, Y)+(s-t) \Delta(\mathbb{A}),
\end{aligned}
$$

which implies that $d(X, Y) \leq \frac{s-t}{s} \Delta(\mathbb{A})$.

Step 3. $d\left(P_{t}(\omega ; \mathbb{A}), P_{t}(\mu ; \mathbb{A})\right) \leq \frac{1}{t} d(\omega, \mu)$.

Let $X=P_{t}(\omega ; \mathbb{A})$ and $Y=P_{t}(\mu ; \mathbb{A})$. Then

$$
\begin{aligned}
d(X, Y) & =d\left(\sum_{i=1}^{n} w_{i}\left(X \#_{t} A_{i}\right), \sum_{i=1}^{n} u_{i}\left(Y \#_{t} A_{i}\right)\right) \\
& =d\left(\sum_{i=1}^{n} X \#_{t}\left(w_{i}^{1 / t} A_{i}\right), \sum_{i=1}^{n} Y \#_{t}\left(u_{i}^{1 / t} A_{i}\right)\right) \\
& \leq \max _{1 \leq i \leq n} d\left(X \#_{t}\left(w_{i}^{1 / t} A_{i}\right), Y \#_{t}\left(u_{i}^{1 / t} A_{i}\right)\right) \\
& \leq \max _{1 \leq i \leq n}\left[(1-t) d(X, Y)+t d\left(w_{i}^{1 / t} A_{i}, u_{i}^{1 / t} A_{i}\right)\right] \\
& =(1-t) d(X, Y)+\max _{1 \leq i \leq n}\left|\log w_{i}-\log u_{i}\right|=(1-t) d(X, Y)+d(\omega, \mu)
\end{aligned}
$$


Step 4. $d\left(P_{t}(\omega ; \mathbb{A}), P_{t}(\omega ; \mathbb{B})\right) \leq \max _{1 \leq i \leq n} d\left(A_{i}, B_{i}\right)$.

Let $X=P_{t}(\omega ; \mathbb{A})$ and $Y=P_{t}(\mu ; \mathbb{B})$. Then from Lemmas $2.2(\mathrm{i})$,(iii) and 2.4

$$
\begin{aligned}
d(X, Y) & =d\left(\sum_{i=1}^{n} w_{i}\left(X \#_{t} A_{i}\right), \sum_{i=1}^{n} w_{i}\left(Y \#_{t} B_{i}\right)\right) \\
& \leq \max _{1 \leq i \leq n} d\left(X \#_{t} A_{i}, Y \#_{t} B_{i}\right) \leq \max _{1 \leq i \leq n}\left[(1-t) d(X, Y)+t d\left(A_{i}, B_{i}\right)\right] \\
& =(1-t) d(X, Y)+t \max _{1 \leq i \leq n} d\left(A_{i}, B_{i}\right) .
\end{aligned}
$$

By the triangular inequality and Steps 2-4,

$$
\begin{aligned}
d\left(P_{t}(\omega ; \mathbb{A}), P_{s}(\mu ; \mathbb{B})\right) \leq & d\left(P_{t}(\omega ; \mathbb{A}), P_{s}(\omega ; \mathbb{A})\right)+d\left(P_{s}(\omega ; \mathbb{A}), P_{s}(\mu ; \mathbb{A})\right) \\
& +d\left(P_{s}(\mu ; \mathbb{A}), P_{s}(\mu ; \mathbb{B})\right) \\
\leq & \frac{s-t}{s} \Delta(\mathbb{A})+\frac{1}{s} d(\omega, \mu)+\max _{1 \leq i \leq n} d\left(A_{i}, B_{i}\right)
\end{aligned}
$$

for $0<t \leq s \leq 1$

For $\mathbb{A}=\left(A_{1}, \ldots, A_{n}\right) \in \mathbb{P}^{n}, M \in \operatorname{GL}(H), \mathbf{a}=\left(a_{1}, \ldots, a_{n}\right) \in(0, \infty)^{n}, \omega=$ $\left(w_{1}, \ldots, w_{n}\right) \in \Delta_{n}$, and for a permutation $\sigma$ on $n$-letters, we set

$$
\begin{aligned}
M \mathbb{A} M^{*} & =\left(M A_{1} M^{*}, \ldots, M A_{n} M^{*}\right), \quad \mathbb{A}_{\sigma}=\left(A_{\sigma(1)}, \ldots, A_{\sigma_{\sigma(n)}}\right), \\
\mathbb{A}^{(k)} & =(\underbrace{\mathbb{A}, \ldots, \mathbb{A}}_{k}) \in \mathbb{P}^{n k}, \omega^{(k)}=\frac{1}{k}(\underbrace{\omega, \ldots, \omega}_{k}) \in \Delta_{n k}, \\
\mathbf{a}^{t} & =\left(a_{1}^{t}, \ldots, a_{n}^{t}\right), \omega \odot \mathbf{a}=\frac{1}{\sum_{i=1}^{n} w_{i} a_{i}}\left(w_{1} a_{1}, \ldots, w_{n} a_{n}\right) \in \Delta_{n}, \\
\hat{\omega} & =\frac{1}{1-w_{n}}\left(w_{1}, \ldots, w_{n-1}\right) \in \Delta_{n-1}, \quad \mathbf{a} \cdot \mathbb{A}=\left(a_{1} A_{1}, \ldots, a_{n} A_{n}\right) .
\end{aligned}
$$

We list some basic properties of $P_{t}(\omega ; \mathbb{A})$.

Proposition 3.6. Let $\mathbb{A}=\left(A_{1}, \ldots, A_{n}\right), \mathbb{B}=\left(B_{1}, \ldots, B_{n}\right) \in \mathbb{P}^{n}, \omega \in \Delta_{n}, \mathbf{a}=$ $\left(a_{1}, \ldots, a_{n}\right) \in(0, \infty)^{n}$ and let $s, t \in[-1,1] \backslash\{0\}$.

(1) $P_{t}(\omega ; \mathbb{A})=\left(\sum_{i=1}^{n} w_{i} A_{i}^{t}\right)^{\frac{1}{t}}$ if the $A_{i}$ 's commute;

(2) $P_{t}(\omega ; \mathbf{a} \cdot \mathbb{A})=\left(\sum_{i=1}^{n} w_{i} a_{i}^{t}\right)^{\frac{1}{t}} P_{t}\left(\omega \odot \mathbf{a}^{t} ; \mathbb{A}\right)$;

(3) $P_{t}\left(\omega_{\sigma} ; \mathbb{A}_{\sigma}\right)=P_{t}(\omega ; \mathbb{A})$ for any permutation $\sigma ;$

(4) $P_{t}(\omega ; \mathbb{A}) \leq P_{t}(\omega ; \mathbb{B})$ if $A_{i} \leq B_{i}$ for all $i=1,2, \ldots, n$;

(5) $d\left(P_{t}(\omega ; \mathbb{A}), P_{t}(\omega ; \mathbb{B})\right) \leq \max _{1 \leq i \leq n}\left\{d\left(A_{i}, B_{i}\right)\right\}$;

(6) $(1-u) P_{|t|}(\omega ; \mathbb{A})+u P_{|t|}(\omega ; \mathbb{B}) \leq P_{|t|}(\omega ;(1-u) \mathbb{A}+u \mathbb{B})$ for any $u \in[0,1]$;

(7) $P_{t}\left(\omega ; M \mathbb{A} M^{*}\right)=M P_{t}(\omega ; \mathbb{A}) M^{*}$ for any invertible $M$;

(8) $P_{t}\left(\omega ; \mathbb{A}^{-1}\right)^{-1}=P_{-t}(\omega ; \mathbb{A})$;

(9) $\left(\sum_{i=1}^{n} w_{i} A_{i}^{-1}\right)^{-1} \leq P_{t}(\omega ; \mathbb{A}) \leq \sum_{i=1}^{n} w_{i} A_{i}$;

(10) $P_{t}\left(\omega^{(k)} ; \mathbb{A}^{(k)}\right)=P_{t}(\omega ; \mathbb{A})$ for any $k \in \mathbb{N}$;

(11) $P_{t}\left(\omega ; A_{1}, \ldots, A_{n-1}, X\right)=X$ if and only if $X=P_{t}\left(\hat{\omega} ; A_{1}, \ldots, A_{n-1}\right)$. In particular, $P_{t}\left(A_{1}, \ldots, A_{n}, X\right)=X$ if and only if $X=P_{t}\left(A_{1}, \ldots, A_{n}\right)$;

(12) for $s \in(0,1], P_{t}\left(\omega ; X \#_{s} A_{1}, \ldots, X \#_{s} A_{n}\right)=X$ if and only if $X=P_{s t}(\omega ; \mathbb{A})$;

(13) if $t \in(0,1]$, then $\Phi\left(P_{t}(\omega ; \mathbb{A})\right) \leq P_{t}(\omega ; \Phi(\mathbb{A}))$ for any positive unital linear map $\Phi$, where $\Phi(\mathbb{A})=\left(\Phi\left(A_{1}\right), \ldots, \Phi\left(A_{n}\right)\right)$. 
Proof. Item (5) was shown in Step 4 of the previous proposition. We provide a proof of (4). The other proofs are similar to those of [17.

Suppose that $A_{i} \leq B_{i}$ for all $i=1,2, \ldots, n$. Let $t \in(0,1]$. Define $f(X)=$ $\sum_{i=1}^{n} w_{i}\left(X \#_{t} A_{i}\right)$ and $g(X)=\sum_{i=1}^{n} w_{i}\left(X \#_{t} B_{i}\right)$. Then $P_{t}(\omega ; \mathbb{A})=\lim _{k \rightarrow \infty} f^{k}(X)$ and $P_{t}(\omega ; \mathbb{B})=\lim _{k \rightarrow \infty} g^{k}(X)$ for any $X \in \mathbb{P}$, by the Banach fixed point theorem. By the Loewner-Heinz inequality, $f(X) \leq g(X)$ for all $X \in \mathbb{P}$, and $f(X) \leq$ $f(Y), g(X) \leq g(Y)$ whenever $X \leq Y$. Let $X_{0}>0$. Then $f\left(X_{0}\right) \leq g\left(X_{0}\right)$ and $f^{2}\left(X_{0}\right)=f\left(f\left(X_{0}\right)\right) \leq g\left(f\left(X_{0}\right)\right) \leq g^{2}\left(X_{0}\right)$. Inductively, we have $f^{k}\left(X_{0}\right) \leq g^{k}\left(X_{0}\right)$ for all $k \in \mathbb{N}$. Therefore, $P_{t}(\omega ; \mathbb{A})=\lim _{k \rightarrow \infty} f^{k}\left(X_{0}\right) \leq \lim _{k \rightarrow \infty} g^{k}\left(X_{0}\right)=P_{t}(\omega ; \mathbb{B})$.

Let $t \in[-1,0)$. Then $\mathbb{A}^{-1} \geq \mathbb{B}^{-1}$ and thus $P_{-t}\left(\omega ; \mathbb{A}^{-1}\right) \geq P_{-t}\left(\omega ; \mathbb{B}^{-1}\right)$. Therefore, $P_{t}(\omega ; \mathbb{A})=P_{-t}\left(\omega ; \mathbb{A}^{-1}\right)^{-1} \leq P_{-t}\left(\omega ; \mathbb{B}^{-1}\right)^{-1}=P_{t}(\omega ; \mathbb{B})$.

Remark 3.7. By Proposition 3.6 (2), the power mean $P_{t}(\omega ; \cdot): \mathbb{P}^{n} \rightarrow \mathbb{P}$ is homogeneous $P_{t}\left(\omega ; a A_{1}, \ldots, a A_{n}\right)=a P_{t}\left(\omega ; A_{1}, \ldots, A_{n}\right)$ and by $(4)$ it is monotonic.

\section{THE POWER MEAN LIMIT}

In [17] Lim and Pálfia have shown in the finite-dimensional setting that the Karcher or least-squares mean is the limit as $t \rightarrow 0^{+}$of the (monotonically decreasing) family of power means $P_{t}$. We take this characterization as the launch point for our approach to the infinite-dimensional Karcher mean.

We recall that the strong topology on the space $\mathcal{B}(H)$ of bounded linear operators is the topology of pointwise convergence. If a net of positive semidefinite operators $A_{\alpha}$ converges strongly to $A$, then the non-negative values $\left\langle x, A_{\alpha} x\right\rangle$ must converge to a non-negative $\langle x, A x\rangle$, so the cone $\{A: 0 \leq A\}$ is strongly closed. Hence the partial order $\{(A, B) \in \mathcal{S}(H) \times \mathcal{S}(H): A \leq B\}$ is strongly closed, since $A_{\alpha} \leq B_{\alpha}$ and $A_{\alpha} \rightarrow A, B_{\alpha} \rightarrow B$ in the strong topology imply $B_{\alpha}-A_{\alpha} \geq 0$ strongly converges to $B-A \geq 0$. We also recall the well-known fact that any monotonically decreasing net of self-adjoint operators that is bounded below possesses an infimum $A$ to which it strongly converges (see, for example, Theorem 4.28(b) of [23]). Dually a monotonically increasing net that is bounded above strongly converges to its supremum.

For $G, H: \Delta_{n} \times \mathbb{P}^{n} \rightarrow \mathbb{P}$, we define $G \leq H$ if $G(\omega ; \mathbb{A}) \leq H(\omega ; \mathbb{A})$ for all $\omega \in \Delta_{n}$ and $\mathbb{A} \in \mathbb{P}^{n}$. We note that $\mathcal{H} \leq \mathcal{A}$, the arithmetic-harmonic mean inequality.

Theorem 4.1. Let $\omega \in \Delta_{n}$ and $\mathbb{A} \in \mathbb{P}^{n}$. Then there exist $X_{ \pm} \in \mathbb{P}$ such that

$$
\lim _{t \rightarrow 0^{ \pm}} P_{t}(\omega ; \mathbb{A})=X_{ \pm}
$$

under the strong-operator topology. Define $P_{0^{ \pm}}(\omega ; \mathbb{A})=X_{ \pm}$. Then for $0<t \leq s \leq$ 1 ,

$$
\mathcal{H}=P_{-1} \leq P_{-s} \leq P_{-t} \leq \cdots \leq P_{0^{-}} \leq P_{0^{+}} \leq \cdots \leq P_{t} \leq P_{s} \leq \mathcal{A} .
$$

Proof. Let $\omega=\left(w_{1}, \ldots, w_{n}\right) \in \Delta_{n}$ and $\mathbb{A}=\left(A_{1}, \ldots, A_{n}\right) \in \mathbb{P}^{n}$.

Step 1. $P_{t} \leq P_{s} \leq P_{1}=\mathcal{A}$ for $0<t \leq s \leq 1$.

Let $f: \mathbb{P} \rightarrow \mathbb{P}$ be defined by $f(X)=\sum_{i=1}^{n} w_{i}\left(X \#_{t} A_{i}\right)$. By the Banach fixed point theorem, $P_{t}(\omega ; \mathbb{A})=\lim _{k \rightarrow \infty} f^{k}(X)$ for any $X \in \mathbb{P}$. We observe from the fact 
$X=X \#_{0} A_{i}$ and Lemma 2.1, parts (vii) and (viii), that

$$
\begin{aligned}
f(X) & =\sum_{i=1}^{n} w_{i}\left(X \#_{t} A_{i}\right)=\sum_{i=1}^{n} w_{i}\left[X \#_{\frac{t}{s}}\left(X \#_{s} A_{i}\right)\right] \\
& \leq \sum_{i=1}^{n} w_{i}\left[\left(1-\frac{t}{s}\right) X+\frac{t}{s}\left(X \#_{s} A_{i}\right)\right] \\
& =\left(1-\frac{t}{s}\right) X+\frac{t}{s} \sum_{i=1}^{n} w_{i}\left(X \#_{s} A_{i}\right) .
\end{aligned}
$$

Applying the preceding to $X_{0}=P_{s}(\omega ; \mathbb{A})$ yields

$$
f\left(X_{0}\right) \leq\left(1-\frac{t}{s}\right) X_{0}+\frac{t}{s} \sum_{i=1}^{n} w_{i}\left(X_{0} \#_{s} A_{i}\right)=\left(1-\frac{t}{s}\right) X_{0}+\frac{t}{s} X_{0}=X_{0} .
$$

Since $f$ is monotonic (Remark 3.4),$f^{k+1}\left(X_{0}\right) \leq f^{k}\left(X_{0}\right) \leq \cdots \leq f\left(X_{0}\right) \leq X_{0}$ for all $k \in \mathbb{N}$. Therefore, $P_{t}(\omega ; \mathbb{A})=\lim _{k \rightarrow \infty} f^{k}\left(X_{0}\right) \leq X_{0}=P_{s}(\omega ; \mathbb{A})$.

Step 2. $P_{-t} \leq P_{t}$ for $0<t \leq 1$. Let $X=P_{t}(\omega ; \mathbb{A})$ and let $Y=P_{-t}(\omega ; \mathbb{A})$. Then by definition of $P_{-t}, Y=P_{t}\left(\omega ; \mathbb{A}^{-1}\right)^{-1}$ and $Y^{-1}=\sum_{i=1}^{n} w_{i}\left(Y^{-1} \#_{t} A_{i}^{-1}\right)=$ $\sum_{i=1}^{n} w_{i}\left(Y \#_{t} A_{i}\right)^{-1}$. We consider the map $f(Z)=\left[\sum_{i=1}^{n} w_{i}\left(Z \#_{t} A_{i}\right)^{-1}\right]^{-1}$. Then

$$
f(X)=\left[\sum_{i=1}^{n} w_{i}\left(X \#_{t} A_{i}\right)^{-1}\right]^{-1} \leq \sum_{i=1}^{n} w_{i}\left(X \#_{t} A_{i}\right)=X .
$$

Since $f$ is monotonic, $f^{k}(X) \leq X$ for all $k \in \mathbb{N}$. By Remark 3.4,

$$
P_{-t}(\omega ; \mathbb{A})=Y=\lim _{k \rightarrow \infty} f^{k}(X) \leq X=P_{t}(\omega ; \mathbb{A}) .
$$

Step 3. $\mathcal{H}=P_{-1} \leq P_{-s} \leq P_{-t}$ for $0<t \leq s \leq 1$.

Let $X=P_{-s}(\omega ; \mathbb{A})$ and $Y=P_{-t}(\omega ; \mathbb{A})$. By Step $1, X^{-1}=P_{s}\left(\omega ; \mathbb{A}^{-1}\right) \geq$ $P_{t}\left(\omega ; \mathbb{A}^{-1}\right)=Y^{-1}$. Thus, $X \leq Y$.

Step 4. $\mathcal{H}=P_{-1} \leq P_{-s} \leq P_{-t} \leq \cdots \leq P_{t} \leq P_{s} \leq \mathcal{A}, \quad 0<t \leq s \leq 1$. Follows from Steps 1-3.

Finally the nets $\left\{P_{t}(\omega ; \mathbb{A})\right\}_{t>0}$ and $\left\{P_{-t}(\omega ; \mathbb{A})\right\}_{t>0}$ are monotonic and bounded between $\mathcal{H}(\omega ; \mathbb{A})$ and $\mathcal{A}(\omega ; \mathbb{A})$. Therefore, there exist $X_{ \pm} \in \mathbb{P}$ such that

$$
\lim _{t \rightarrow 0^{+}} P_{t}(\omega ; \mathbb{A})=X_{+}, \quad \lim _{t \rightarrow 0^{-}} P_{t}(\omega ; \mathbb{A})=X_{-}
$$

under the strong-operator topology. By Step $2, P_{t} \geq P_{-t}$ for all $t \in(0,1]$. Since the partial order on $\mathcal{S}(H)$ is strongly closed, their strong limits satisfy $X_{+} \geq X_{-}$.

Remark 4.2. The monotonically decreasing property $P_{t} \leq P_{s},-1 \leq t \leq s \leq 1$, is new, even for the finite-dimensional setting considered by Lim and Pálfia [17.

Definition 4.3. We set $\Lambda\left(\omega ; A_{1}, \ldots, A_{n}\right)=P_{0^{+}}\left(\omega ; A_{1}, \ldots, A_{n}\right)$ and call it the $\omega$ weighted Karcher mean of $A_{1}, \ldots, A_{n}$. We set $\Lambda^{*}\left(\omega ; A_{1}, \ldots, A_{n}\right)=P_{0^{-}}\left(\omega ; A_{1}, \ldots, A_{n}\right)$.

The basic properties of power means in Proposition 3.6 together with Theorem 4.1 provide some important properties of the Karcher mean.

Theorem 4.4. (P1) (Consistency with scalars) $\Lambda(\omega ; \mathbb{A})=A_{1}^{w_{1}} \cdots A_{n}^{w_{n}}$ if the $A_{i}$ 's commute;

(P2) (Homogeneity) $\Lambda\left(\omega ; a A_{1}, \ldots, a A_{n}\right)=a \Lambda(\omega ; \mathbb{A})$; 
(P3) (Permutation invariance) $\Lambda\left(\omega_{\sigma} ; \mathbb{A}_{\sigma}\right)=\Lambda(\omega ; \mathbb{A})$;

(P4) (Monotonicity) if $B_{i} \leq A_{i}$ for all $1 \leq i \leq n$, then $\Lambda(\omega ; \mathbb{B}) \leq \Lambda(\omega ; \mathbb{A})$;

(P5) (Continuity) $d(\Lambda(\omega ; \mathbb{A}), \Lambda(\omega ; \mathbb{B})) \leq \max _{1 \leq i \leq n}\left\{d\left(A_{i}, B_{i}\right)\right\}$ for the Thompson metric d; in particular $\Lambda(\omega, \cdot)$ is continuous;

(P6) (Invariancy) $\Lambda\left(\omega ; M^{*} \mathbb{A} M\right)=M^{*} \Lambda(\omega ; \mathbb{A}) M$ for any invertible $M$;

(P7) (Joint concavity) $\Lambda(\omega ;(1-u) \mathbb{A}+u \mathbb{B}) \geq(1-u) \Lambda(\omega ; \mathbb{A})+u \Lambda(\omega ; \mathbb{B})$ for $0 \leq u \leq 1$

(P8) (Duality) $\Lambda\left(\omega ; A_{1}^{-1}, \ldots, A_{n}^{-1}\right)^{-1}=\Lambda^{*}\left(\omega ; A_{1}, \ldots, A_{n}\right)$;

(P9) (AGH weighted mean inequalities) $\left(\sum_{i=1}^{n} w_{i} A_{i}^{-1}\right)^{-1} \leq \Lambda(\omega ; \mathbb{A}) \leq \sum_{i=1}^{n} w_{i} A_{i}$.

Proof. (P1) Follows from the fact that $\left(\sum_{i=1}^{n} w_{i} A_{i}^{t}\right)^{\frac{1}{t}}$ converges to $\exp \left(\sum_{i=1}^{n} w_{i} \log A_{i}\right)$ with respect to the operator norm (see e.g. [19]) and by Proposition [3.6 and Theorem 4.1 ,

(P2) Let $a>0$. Then

$$
\begin{aligned}
P_{0^{+}}\left(\omega ; a A_{1}, \ldots, a A_{n}\right) x & =\lim _{t \rightarrow 0^{+}} P_{t}\left(\omega ; a A_{1}, \ldots, a A_{n}\right) x=\lim _{t \rightarrow 0^{+}}\left[a P_{t}\left(\omega ; A_{1}, \ldots, A_{n}\right) x\right] \\
& =a \lim _{t \rightarrow 0^{+}} P_{t}\left(\omega ; A_{1}, \ldots, A_{n}\right) x=a P_{0^{+}}\left(\omega ; A_{1}, \ldots, A_{n}\right) x .
\end{aligned}
$$

(P5). Let $X_{t}=P_{t}(\omega ; \mathbb{A})$ and $Y_{t}=P_{t}(\omega ; \mathbb{B})$. Let $\alpha:=\max _{1 \leq i \leq n}\left\{d\left(A_{i}, B_{i}\right)\right\}$. By Proposition 3.5. $d\left(X_{t}, Y_{t}\right) \leq \alpha$ for the Thompson metric $d$ and thus $X_{t} \leq e^{\alpha} Y_{t}$ for $0<t \leq 1$. Since $\Lambda(\omega ; \mathbb{A}) \leq X_{t}$ for each $t$, we have $\Lambda(\omega ; \mathbb{A}) \leq e^{\alpha} Y_{t}$. By the strong closedness of the order and the strong convergence of $Y_{t}$ to $\Lambda(\omega ; \mathbb{B})$ as $t \rightarrow 0^{+}$, $\Lambda(\omega ; \mathbb{A}) \leq e^{\alpha} \Lambda(\omega ; \mathbb{B})$. Similarly $\Lambda(\omega ; \mathbb{B}) \leq e^{\alpha} \Lambda(\omega ; \mathbb{A})$. It follows from definition of the Thompson metric that $d(\Lambda(\omega ; \mathbb{A}), \Lambda(\omega ; \mathbb{B})) \leq \alpha=\max \left\{d\left(A_{i}, B_{i}\right): 1 \leq i \leq n\right\}$.

(P8). Follows from Proposition 3.6 (8),(9) and the strong continuity of inversion on bounded intervals (see Lemma 5.4).

By Proposition 3.6. Theorem 4.1 and the strong closedness of the order, the remaining parts of the proof are immediate.

Remark 4.5. We note that $P_{0^{-}}(\omega ; \mathbb{A})$ satisfies all the preceding properties except the joint concavity. For a finite-dimensional Hilbert space, $P_{0^{-}}=\Lambda$ (see [17]) and the Karcher mean $\Lambda(\omega ; \mathbb{A})$ is the unique solution of the Karcher equation

$$
\sum_{i=1}^{n} w_{i} \log \left(X^{-1 / 2} A_{i} X^{-1 / 2}\right)=0 .
$$

Then, via the Karcher equation, the Karcher mean satisfies the joint homogeneity property $([18]) \Lambda\left(\omega ; a_{1} A_{1}, \ldots, a_{n} A_{n}\right)=a_{1}^{w_{1}} \cdots a_{n}^{w_{n}} \Lambda(\omega ; \mathbb{A})$ and the contraction property $\delta(\Lambda(\omega ; \mathbb{A}), \Lambda(\omega ; \mathbb{B})) \leq \sum_{i=1}^{n} w_{i} \delta\left(A_{i}, B_{i}\right)$ with respect to the Riemannian trace metric $\delta(A, B)=\left[\sum_{i=1}^{m} \log ^{2} \lambda_{i}\left(A^{-1} B\right)\right]^{\frac{1}{2}}$, where the $\lambda_{i}(X)$ denote the eigenvalues of $X$. By Proposition 2.5, the contraction property holds for the Thompson metric. We eventually extend these results to the infinite-dimensional setting; see Theorem 6.8

\section{THE KARCHER EQUATION}

Let $\mathbb{A}=\left(A_{1}, \ldots, A_{n}\right) \in \mathbb{P}^{n}, \omega=\left(w_{1}, \ldots, w_{n}\right) \in \Delta_{n}$. We consider the following non-linear operator equation on $\mathbb{P}$, called the Karcher equation:

$$
\sum_{i=1}^{n} w_{i} \log \left(X^{-1 / 2} A_{i} X^{-1 / 2}\right)=0 .
$$


Note that multiplying by -1 yields the equivalent equation

$$
\sum_{i=1}^{n} w_{i} \log \left(X^{1 / 2} A_{i}^{-1} X^{1 / 2}\right)=0,
$$

and we pass freely between the two.

In the finite-dimensional setting it is known that the least-squares mean (the Karcher mean) satisfies the Karcher equation, indeed is the unique positive solution of the Karcher equation (cf. Remark 4.5). Our goal in this section is to show that the Karcher mean we have defined in the preceding section satisfies the Karcher equation (and hence is aptly named).

Let $\log z$ denote the principal branch of the complex logarithm defined on $\mathbb{C} \backslash$ $(-\infty, 0]$ defined by $\log z=\ln |z|+i \operatorname{Arg} z$, where $\operatorname{Arg} z$ is the principal branch of the argument taking values in $(-\pi, \pi]$. By the holomorphic functional calculus $\log A$ is defined for any bounded linear operator $A$ with spectrum contained in $\mathbb{C} \backslash(-\infty, 0]$, in particular with spectrum contained in $(0, \infty)$. We recall the following standard fact.

Lemma 5.1. For $\mathbb{A}=\left(A_{1}, \ldots, A_{n}\right) \in \mathbb{P}^{n}, X$ satisfies the Karcher equation if and only if it satisfies

$$
\sum_{i=1}^{n} w_{i} \log \left(A_{i}^{-1} X\right)=0 .
$$

Proof. We note that $X^{-1 / 2}\left(X^{-1 / 2} A_{i} X^{-1 / 2}\right) X^{1 / 2}=X^{-1} A_{i}$, so $X^{-1 / 2} A_{i} X^{-1 / 2}$ is similar to $X^{-1} A_{i}$, and hence the latter has positive spectrum and thus a logarithm. Applying inner automorphism by $X^{-1 / 2}$ to equation (5.7) then yields the equation

$$
\sum_{i=1}^{n} w_{i} \log \left(X^{-1} A_{i}\right)=0
$$

and applying inner automorphism by $X^{1 / 2}$ to equation (5.9) yields equation (5.7). Hence $X$ satisfies equation (5.7) if and only if (5.9). Now multiplying equation (5.9) by -1 yields (5.8) and vice versa; hence the two have the same solution sets, and thus so do (5.7) and (5.8).

Lemma 5.2. Operator multiplication is strongly jointly continuous when restricted to any bounded subset.

Proof. If in the set of all operators with norm bounded by $M, A_{\alpha} \rightarrow A$ strongly and $B_{\alpha} \rightarrow B$ strongly, then

$\left\|\left(A_{\alpha} B_{\alpha}-A B\right) x\right\| \leq\left\|A_{\alpha}\left(B_{\alpha}-B\right) x\right\|+\left\|\left(A_{\alpha}-A\right) B x\right\| \leq M\left\|\left(B_{\alpha}-B\right) x\right\|+\left\|\left(A_{\alpha}-A\right) B x\right\|$, where the last two terms go to 0 by definition of strong convergence.

The following lemma is a special case of Theorem 3.6 from Kadison's study of strongly continuous operator functions [9] on self-adjoint operators.

Lemma 5.3. Let $Q$ be an open or closed subset of $\mathbb{R}$ and let $f: Q \rightarrow \mathbb{R}$ be a continuous bounded function. Then the corresponding operator function $\hat{f}$ is strongoperator continuous on the set $\mathcal{S} H_{Q}$ of bounded self-adjoint operators on a Hilbert space $H$ with spectra in $Q$. 
Lemma 5.4. The following functions are strongly continuous on $\left[e^{-m} I, e^{m} I\right]=$ $\left\{A \in \mathcal{S}(H): e^{-m} I \leq A \leq e^{m} I\right\}$.

(i) The logarithm map $A \mapsto \log A$, which is also monotonic.

(ii) The power map $A \mapsto A^{r}$ for $-1 \leq r \leq 1$.

(iii) The binary weighted mean map $(A, B) \mapsto A \#_{t} B$ for $0 \leq t \leq 1$.

The last two functions have image contained in $\left[e^{-m} I, e^{m} I\right]$.

Proof. Let $A \in\left[e^{-m} I, e^{m} I\right]$. For $r<e^{-m}$, we have $r I<e^{-m} I$ and so $0 \leq$ $A-e^{-m} I<A-r I$. This shows that $A-r I \in \mathbb{P}$, hence invertible, and hence $r \notin \operatorname{Spec}(A)$. In a similar fashion one sees that $r>e^{m}$ is not in the spectrum of $A$, hence $\operatorname{spec}(A) \subseteq\left[e^{-m}, e^{m}\right]$. Since $\log (x)$ and $x^{r}$ are continuous and bounded on the bounded set $\left[e^{-m}, e^{m}\right]$, we conclude from Lemma 5.3 that the corresponding operator functions are strongly continuous when restricted to $\left[e^{-m} I, e^{m} I\right]$. It is well known that $\log$ is monotonic on $\mathbb{P}$.

For $A \in\left[e^{-m} I, e^{m} I\right]$ and $0 \leq r \leq 1, e^{-m} I \leq\left(e^{-m}\right)^{r} I \leq A^{r} \leq\left(e^{m}\right)^{r} I \leq e^{m} I$ by the Loewner-Heinz inequality. Since inversion is order-reversing, $\left[e^{-m} I, e^{m} I\right]$ is stable under inversion, and it follows that also $A^{-r} \in\left[e^{-m} I, e^{m} I\right]$.

For $A \in\left[e^{-m} I, e^{m} I\right],\|A\| \leq e^{m}$ by Lemma 2.3(ii). Since

$$
A \#{ }_{t} B=A^{1 / 2}\left(A^{-1 / 2} B A^{-1 / 2}\right)^{t} A^{1 / 2},
$$

$0 \leq t \leq 1$, and all partial steps in the computation are bounded in norm by $e^{3 m}$ (since $A^{ \pm 1 / 2} \leq e^{m / 2} I$ ) for $A, B \in\left[e^{-m} I, e^{m} I\right]$, the operation is strongly continuous by Lemma 5.2 and part (ii). Since the weighted mean is monotonic and idempotent, $e^{-m} I=e^{-m} I \#_{t} e^{-m} \leq A \#_{t} B \leq e^{m} I \#_{t} e^{m} I=e^{m} I$.

Lemma 5.5. Let $V \in S(H)$. Then on any ball $B_{m}(0)=\{U \in S(H):\|U\| \leq m\}$ such that $\|V\|<m$

$$
\lim _{(t, U) \rightarrow(0, V)} \frac{e^{t U}-I}{t}=V,
$$

where the limit is taken in the strong-operator topology.

Proof. Let $U \in B_{m}(0)$. Then

$$
\begin{aligned}
\left\|\frac{e^{t U}-I}{t}-U\right\| & =\left\|\frac{\sum_{k=0}^{\infty} \frac{1}{k !}(t U)^{k}-I}{t}-U\right\| \\
& =\left\|\sum_{k=1}^{\infty} \frac{1}{k !} t^{k-1} U^{k}-U\right\|=\left\|\sum_{k=2}^{\infty} \frac{t^{k-1}}{k !} U^{k}\right\| \\
& \leq \sum_{k=2}^{\infty} \frac{t^{k-1}}{k !}\left\|U^{k}\right\| \leq \sum_{k=2}^{\infty} \frac{t^{k-1}}{k !}\|U\|^{k} \\
& \leq\|U\|\left[e^{t\|U\|}-1\right] \leq m\left(e^{t m}-1\right) .
\end{aligned}
$$

Let $x \in H$. Then as $t \rightarrow 0$ and $U \rightarrow V$ strongly,

$$
\begin{aligned}
\left\|\frac{e^{t U}-I}{t} x-V x\right\| & \leq\left\|\frac{e^{t U}-I}{t} x-U x\right\|+\|U x-V x\| \\
& \leq m\left(e^{t m}-1\right)\|x\|+\|U x-V x\| \rightarrow 0 .
\end{aligned}
$$


The following shows that the Karcher mean is a solution of the Karcher equation.

Theorem 5.6. For each $\omega \in \Delta_{n}$ and $\mathbb{A} \in \mathbb{P}^{n}, \Lambda(\omega ; \mathbb{A})$ satisfies the Karcher equation (5.7).

Proof. Let $t \in(0,1]$. Let $X_{t}=P_{t}(\omega ; \mathbb{A})$ and let $X_{0}=\Lambda(\omega ; \mathbb{A})=P_{0^{+}}(\omega ; \mathbb{A})$. By Theorem 4.1, with respect to the strong topology $X_{t} \rightarrow X_{0}$ monotonically as $t \rightarrow 0^{+}$ and $X_{0}=\Lambda(\omega ; \mathbb{A}) \leq X_{t} \leq P_{1}(\omega ; \mathbb{A})=X_{1}$ for all $t \in[0,1]$. Pick $m$ such that $A_{i}, X_{1}, X_{0} \in\left[e^{-m / 2} I, e^{m / 2} I\right]$ for all $i$. It follows that $X_{t} \in\left[e^{-m / 2} I, e^{m / 2} I\right]$ for all $0 \leq t \leq 1$.

By the order reversal of inversion $\left[e^{-m / 2} I, e^{m / 2} I\right]$ is closed under inversion so that

$$
X_{t}^{-1 / 2} A_{i} X_{t}^{-1 / 2} \leq X_{t}^{-1 / 2}\left(e^{m / 2} I\right) X_{t}^{-1 / 2}=e^{m / 2} X_{t}^{-1} \leq e^{m} I
$$

and similarly $e^{-m} I \leq X_{t}^{-1 / 2} A_{i} X_{t}^{-1 / 2}$. Thus $X_{t}^{-1 / 2} A_{i} X_{t}^{-1 / 2} \in\left[e^{-m} I, e^{m} I\right]$ for all $t \in[0,1]$ and $i=1, \ldots, n$. By part (ii) of Lemma 2.3 we have $\left\|X_{t}^{-1 / 2} A_{i} X_{t}^{-1 / 2}\right\| \leq$ $e^{m}$. By Lemma 5.2 and Lemma [5.4(ii), $X_{t}^{-1 / 2} A_{i} X_{t}^{-1 / 2}$ converges strongly to $X_{0}^{-1 / 2} A_{i} X_{0}^{-1 / 2}$. By strong continuity of $\log$ on $\left[e^{-m} I, e^{m} I\right]$ (Lemma 5.4(i)),

$$
U_{i}:=\log \left(X_{t}^{-1 / 2} A_{i} X_{t}^{-1 / 2}\right) \rightarrow V_{i}:=\log \left(X_{0}^{-1 / 2} A_{i} X_{0}^{-1 / 2}\right)
$$

for all $i$. By Lemma 5.5 applied to any open ball $B_{r}(0), r>e^{m}$, in the strong topology

$$
\lim _{t \rightarrow 0} \frac{\left(X_{t}^{-1 / 2} A_{i} X_{t}^{-1 / 2}\right)^{t}-I}{t}=\lim _{t \rightarrow 0} \frac{e^{t U_{i}}-I}{t}=V_{i}=\log \left(X_{0}^{-1 / 2} A_{i} X_{0}^{-1 / 2}\right)
$$

for all $i=1, \ldots, n$.

By definition, $X_{t}=\sum_{i=1}^{n} w_{i}\left(X_{t} \#_{t} A_{i}\right)$. Pre- and post-multiplying this equation by $X_{t}^{-1 / 2}$ and substituting from equation (2.1) for the weighted mean yields for $t>0$ :

$$
I=\sum_{i=1}^{n} w_{i}\left(X_{t}^{-1 / 2} A_{i} X_{t}^{-1 / 2}\right)^{t},
$$

that is, $0=\sum_{i=1}^{n} w_{i}\left[\frac{\left(X_{t}^{-1 / 2} A_{i} X_{t}^{-1 / 2}\right)^{t}-I}{t}\right]$. By (5.10),

$$
\begin{aligned}
0 & =\lim _{t \rightarrow 0^{+}} \sum_{i=1}^{n} w_{i}\left[\frac{\left(X_{t}^{-1 / 2} A_{i} X_{t}^{-1 / 2}\right)^{t}-I}{t}\right]=\sum_{i=1}^{n} w_{i} \lim _{t \rightarrow 0^{+}}\left[\frac{\left(X_{t}^{-1 / 2} A_{i} X_{t}^{-1 / 2}\right)^{t}-I}{t}\right] \\
& =\sum_{i=1}^{n} w_{i} \log \left(X_{0}^{-1 / 2} A_{i} X_{0}^{-1 / 2}\right) .
\end{aligned}
$$

This shows that $X_{0}=\Lambda(\omega ; \mathbb{A})$ is a solution of the Karcher equation.

Corollary 5.7. The operator $\Lambda^{*}\left(\omega ; A_{1}, \ldots, A_{n}\right)=P_{0^{-}}\left(\omega ; A_{1}, \ldots, A_{n}\right)$ also satisfies the Karcher equation.

Proof. By definition $\Lambda^{*}(\omega ; \mathbb{A})=P_{0^{-}}(\omega ; \mathbb{A})=\lim _{t \rightarrow 0^{-}} X_{t}(\omega ; \mathbb{A}), \mathbb{A}=\left(A_{1}, \ldots, A_{n}\right)$. By strong continuity (or order-reversion) of inversion and the definition of the 
negative power means, $\Lambda^{*}(\omega ; \mathbb{A})^{-1}=\lim _{t \rightarrow 0^{+}} X_{t}\left(\omega ; \mathbb{A}^{-1}\right)=\Lambda\left(\omega ; \mathbb{A}^{-1}\right)$. By the preceding theorem $X=\Lambda\left(\omega ; \mathbb{A}^{-1}\right)$ satisfies the Karcher equation:

$$
\sum_{i=1}^{n} w_{i} \log \left(X^{-1 / 2}\left(A_{i}^{-1}\right) X^{-1 / 2}\right)=0 .
$$

Multiplying the equation by -1 yields

$$
0=\sum_{i=1}^{n} w_{i} \log \left(X^{1 / 2}\left(A_{i}\right) X^{1 / 2}\right)=\sum_{i=1}^{n} w_{i} \log \left(\left(X^{-1}\right)^{-1 / 2} A_{i}\left(X^{-1}\right)^{-1 / 2}\right)=0,
$$

so $\Lambda^{*}(\omega ; \mathbb{A})=X^{-1}$ satisfies the Karcher equation.

\section{Uniqueness of the KarCher MeAN}

For $\omega=\left(w_{1}, \ldots, w_{n}\right) \in \Delta_{n}$ and $\mathbb{A}=\left(A_{1}, \ldots, A_{n}\right) \in \mathbb{P}^{n}$, we consider the corresponding Karcher equation

$$
\sum_{i=1}^{n} w_{i} \log \left(X^{1 / 2} A_{i}^{-1} X^{1 / 2}\right)=0
$$

We denote by $\mathcal{K}(\omega ; \mathbb{A})$ the set of all positive definite solutions $X$ of the Karcher equation (6.11) and consider three important properties of this set.

Lemma 6.1. (1) $\mathcal{K}\left(\omega ; a_{1} A_{1}, \ldots, a_{n} A_{n}\right)=a_{1}^{w_{1}} \cdots a_{n}^{w_{n}} \mathcal{K}(\omega ; \mathbb{A})$ for $a_{1}, \ldots, a_{n}>0$.

(2) $M \mathcal{K}(\omega ; \mathbb{A}) M^{*}=\mathcal{K}\left(\omega ; M \mathbb{A} M^{*}\right)$ for any $M \in \mathrm{GL}(H)$.

Proof. (1) Let $Y=a_{1}^{w_{1}} \cdots a_{n}^{w_{n}} X$. Then

$$
\begin{aligned}
\sum_{i=1}^{n} w_{i} & \log \left(Y^{-1 / 2}\left(a_{i} A_{i}\right) Y^{-1 / 2}\right) \\
& =\sum_{i=1}^{n} w_{i} \log \left(\frac{a_{i}}{\prod_{i=1}^{n} a_{i}^{w_{i}}} X^{-1 / 2} A_{i} X^{-1 / 2}\right) \\
& =\sum_{i=1}^{n} w_{i}\left[\log \left(\frac{a_{i}}{\prod_{i=1}^{n} a_{i}^{w_{i}}} I\right)+\log \left(X^{-1 / 2} A_{i} X^{-1 / 2}\right)\right] \\
& =\log I+\sum_{i=1}^{n} w_{i} \log \left(X^{-1 / 2} A_{i} X^{-1 / 2}\right) \\
& =\sum_{i=1}^{n} w_{i} \log \left(X^{-1 / 2} A_{i} X^{-1 / 2}\right)
\end{aligned}
$$

where the second equality follows from the fact that $\log t A=\log t I+\log A$ for any $t>0$ and $A>0$. Therefore, the left hand side equals 0 iff the right hand side does, which translates to

$$
Y \in \mathcal{K}(\omega ; \mathbf{a} \cdot \mathbb{A})=\mathcal{K}\left(\omega ; a_{1} A_{1}, \ldots, a_{n} A_{n}\right) \text { iff } Y \in a_{1}^{w_{1}} \cdots a_{n}^{w_{n}} \mathcal{K}(\omega ; \mathbb{A}) .
$$

(2) Let $M \in \mathrm{GL}(H)$ and let $M=U P$ be the polar decomposition of $M$, where $U^{*}=U^{-1}$ and $P \in \mathbb{P}$. From $U \log (A) U^{-1}=\log \left(U A U^{-1}\right)$, one computes directly from the Karcher equation as given in Lemma 5.1 that

$$
\mathcal{K}\left(\omega ; U \mathbb{A} U^{*}\right)=U \mathcal{K}(\omega ; \mathbb{A}) U^{*} .
$$


Let $X \in \mathcal{K}(\omega ; \mathbb{A})$. Then $\sum_{i=1}^{n} w_{i} \log \left(A_{i}^{-1} X\right)=0$ (Lemma 5.1) implies that

$$
\begin{aligned}
0 & =P^{-1}\left[\sum_{i=1}^{n} w_{i} \log \left(A_{i}^{-1} X\right)\right] P=\sum_{i=1}^{n} w_{i} \log \left(P^{-1} A_{i}^{-1} X P\right) \\
& =\sum_{i=1}^{n} w_{i} \log \left(\left(P^{-1} A_{i}^{-1} P^{-1}\right)(P X P)\right)
\end{aligned}
$$

and thus $P X P \in \mathcal{K}(\omega ; P \mathbb{A} P)$. This implies that $P \mathcal{K}(\omega ; \mathbb{A}) P \subseteq \mathcal{K}(\omega ; P \mathbb{A} P)$. From $\mathcal{K}(\omega ; \mathbb{A}) \subseteq P^{-1} \mathcal{K}(\omega ; P \mathbb{A} P) P^{-1} \subseteq \mathcal{K}(\omega ; \mathbb{A})$ we conclude that

$$
P \mathcal{K}(\omega ; \mathbb{A}) P=\mathcal{K}(\omega ; P \mathbb{A} P) .
$$

By (6.12) and (6.13), $M \mathcal{K}(\omega ; \mathbb{A}) M^{*}=\mathcal{K}\left(\omega ; M \mathbb{A} M^{*}\right)$.

In the proof of the next theorem we use the following Banach space version of the Implicit Mapping Theorem, taken from [12, Theorem 5.9] and its proof.

Theorem 6.2 (The Implicit Mapping Theorem). Let $U, V$ be open sets in Banach spaces $\mathbf{E}, \mathbf{F}$ respectively, and let $f: U \times V \rightarrow \mathbf{G}$ be a $C^{\infty}$-mapping, where $\mathbf{G}$ is also a Banach space. Let $(a, b) \in U \times V$ and assume that $f(a, b)=0$ and that

$$
D_{2} f(a, b): \mathbf{F} \rightarrow \mathbf{G},
$$

where $D_{2} f$ is the partial with respect to the second variable, is a Banach space isomorphism. Then there exist neighborhoods of $U_{0}$ of $a$ and $V_{0}$ of $b$ and $a C^{\infty}$ map $g: U_{0} \rightarrow V_{0}$ such that $g(a)=b$ and for $(x, y) \in U_{0} \times V_{0}, f(x, y)=0$ if and only if $y=g(x)$.

The Loewner order intervals are defined by $[A, B]:=\{X \in \mathcal{S}(H): A \leq X \leq B\}$ and $(A, B):=\{X: A<X<B\}$.

Theorem 6.3. Let $\omega \in \Delta_{n}$. Then there exists $\epsilon_{\omega}>1$ such that for any $A \in \mathbb{P}$, the Karcher equation (6.11) has a unique solution in $\left[\epsilon_{\omega}^{-1} A, \epsilon_{\omega} A\right]$. Furthermore, the Karcher mean $\Lambda(\omega ; \cdot)$ is $C^{\infty}$ on a neighborhood of the diagonal in $\mathbb{P}^{n}$.

Proof. We consider the map $F^{\omega}: \mathbb{P}^{n} \times \mathbb{P} \rightarrow \mathcal{S}(H)$ by

$$
F^{\omega}\left(A_{1}, \ldots, A_{n}, X\right)=\sum_{i=1}^{n} w_{i} \log \left(X^{1 / 2} A_{i}^{-1} X^{1 / 2}\right),
$$

and for $\mathbb{A}=\left(A_{1}, \ldots, A_{n}\right), F_{\mathbb{A}}^{\omega}(X)=F^{\omega}\left(A_{1}, \ldots, A_{n}, X\right)$. Then $F_{\mathbb{A}}^{\omega}: \mathbb{P} \rightarrow \mathcal{S}(H)$ is $C^{\infty}$, and $F_{\mathbb{A}}^{\omega}(X)=0$ if and only if $X \in \mathcal{K}(\omega ; \mathbb{A})$.

We next employ the elementary differential calculus for open subsets of Banach spaces, as it appears, for example, in [12, Chapter I, $\S 3-5]$. The derivative of $F_{\AA}^{\omega}$ at $X \in \mathbb{P}$ is a bounded linear map from the tangent space at $X$ to the tangent space at $F_{\mathbb{A}}^{\omega}(X)$, which may be considered as a linear map $D F_{\mathbb{A}}^{\omega}(X): \mathcal{S}(H) \rightarrow \mathcal{S}(H)$ since the tangent bundles of $\mathbb{P}$ and $\mathcal{S}(H)$ are trivial with fiber $\mathcal{S}(H)$. Its action is given by

$$
D F_{\mathbb{A}}^{\omega}(X)(Y)=\lim _{t \rightarrow 0} \frac{F_{\mathbb{A}}^{\omega}(X+t Y)-F_{\mathbf{A}}^{\omega}(X)}{t} .
$$

Let $\mathbb{I}=(I, \ldots, I) \in \mathbb{P}^{n}$. Then $F_{\mathbb{I}}^{\omega}(X)=\sum_{i=1}^{n} w_{i} \log X=\log X$, so $F_{\mathbb{I}}^{\omega}(I)=0$ and $D F_{\mathbb{I}}^{\omega}(I)=\operatorname{id}_{\mathcal{S}(H)}$ (which corresponds to and follows from the well-known fact that 
the exponential mapping has derivative the identity at 0 ). Since $F^{\omega}: \mathbb{P}^{n} \times \mathbb{P} \rightarrow$ $\mathcal{S}(H)$ satisfies $F^{\omega}(\mathbb{I}, \cdot)=F_{\mathbb{I}}^{\omega}(\cdot)$, we conclude that the partial derivative of $F^{\omega}$ in the second variable, that is, the $\mathbb{P}$-variable, satisfies

$$
D_{2} F^{\omega}(\mathbb{I}, I)=D F_{\mathbb{I}}^{\omega}(I)=\operatorname{id}_{\mathcal{S}(H)} .
$$

Since $D_{2} F^{\omega}(\mathbb{I}, I)$ is the identity map, in particular a Banach space isomorphism, from $\mathcal{S}(H)$ to $\mathcal{S}(H)$, it follows directly from the Implicit Mapping Theorem (Theorem 6.2) that there exist open neighborhoods $U_{\mathbb{I}}$ of $\mathbb{I}$ in $\mathbb{P}^{n}$ and $V_{I}$ of $I$ in $\mathbb{P}$ and a $C^{\infty}$-mapping $g: U_{\mathbb{I}} \rightarrow V_{I}$ such that $g(\mathbb{I})=I$ and $F^{\omega}(\mathbb{A}, X)=0$ if and only if $X=g(\mathbb{A})$ for all $\mathbb{A} \in U_{\mathbb{I}}, X \in V_{I}$.

Pick $\epsilon>1$ such that $\left[\epsilon^{-1} I, \epsilon I\right]^{n} \subset U_{\mathbb{I}}$ and $\left[\epsilon^{-1} I, \epsilon I\right] \subset V_{I}$. (This is possible since it is easily seen that $\left[\epsilon^{-1} I, \epsilon I\right]$ is the closed $\epsilon$-ball in the Thompson metric.) Then for any $\mathbb{A} \in\left[\epsilon^{-1} I, \epsilon I\right]^{n}$, by the monotonicity and idempotency of $\Lambda$,

$$
\epsilon^{-1} I=\Lambda\left(\omega ; \epsilon^{-1} I, \ldots, \epsilon^{-1} I\right) \leq \Lambda(\omega ; \mathbb{A}) \leq \Lambda(\omega ; \epsilon I, \ldots, \epsilon I)=\epsilon I,
$$

and thus $\Lambda(\omega ; \mathbb{A}) \in V_{I}$.

Since we have shown that the Karcher mean always satisfies the Karcher equation, we have $F^{\omega}(\mathbb{A}, \Lambda(\omega ; \mathbb{A}))=0$, and hence by the preceding two paragraphs $\Lambda(\omega ; \mathbb{A})=g(\mathbb{A})$. Thus the Karcher mean is $C^{\infty}$ on the open neighborhood $\left(\epsilon^{-1} I, \epsilon I\right)^{n}$ of $\mathbb{I}$ and $\mathbb{A} \in\left[\epsilon^{-1} I, \epsilon I\right]^{n}$ has a unique solution to the Karcher equation in $\left[\epsilon^{-1} I, \epsilon I\right]$, namely the Karcher mean $\Lambda(\omega ; \mathbb{A})$.

Next, let $\left(A_{1}, \ldots, A_{n}\right) \in\left[\epsilon^{-1} A, \epsilon A\right]^{n}$. Set $B_{i}=A^{-1 / 2} A_{i} A^{-1 / 2}, i=1, \ldots, n$. Then $B_{i} \in\left[\epsilon^{-1} I, \epsilon I\right]$ for all $i$. By the preceding paragraph, $X=\Lambda(\omega ; \mathbb{B})$ is the unique solution to the Karcher equation $F_{\mathbb{B}}^{\omega}(X)=0$ that lies in $V_{I}$. By Lemma 6.1 and Theorem 4.4

$$
\begin{aligned}
\mathcal{K}\left(\omega ; A_{1}, \ldots, A_{n}\right) \cap\left[\epsilon^{-1} A, \epsilon A\right] & =\mathcal{K}\left(\omega ; A^{1 / 2} B_{1} A^{1 / 2}, \ldots, A^{1 / 2} B_{n} A^{1 / 2}\right) \cap\left[\epsilon^{-1} A, \epsilon A\right] \\
& =A^{1 / 2}\left(\mathcal{K}\left(\omega ; B_{1}, \ldots, B_{n}\right) \cap\left[\epsilon^{-1} I, \epsilon I\right]\right) A^{1 / 2} \\
& =A^{1 / 2}\left\{\Lambda\left(\omega ; B_{1}, \ldots, B_{n}\right)\right\} A^{1 / 2} \\
& =\left\{\Lambda\left(\omega ; A_{1}, \ldots, A_{n}\right)\right\} .
\end{aligned}
$$

This shows that the Karcher equation has the unique solution $\Lambda\left(\omega ; A_{1}, \ldots, A_{n}\right)$ on $\left[\epsilon^{-1} A, \epsilon A\right]$ and is $C^{\infty}$ on the open neighborhood $\left(\epsilon^{-1} A, \epsilon A\right)^{n}$, since

$$
\Lambda\left(\omega ; A_{1}, \ldots, A_{n}\right)=A^{1 / 2} \Lambda\left(\omega ; A^{-1 / 2} A_{1} A^{-1 / 2}, \ldots, A^{-1 / 2} A_{n} A^{-1 / 2}\right) A^{1 / 2} .
$$

Theorem 6.4. The following conditions are equivalent and are all satisfied by $\Lambda$.

(i) $\Lambda: \Delta_{n} \times \mathbb{P}^{n} \rightarrow \mathbb{P}$ is jointly homogeneous;

(ii) $\Lambda: \Delta_{n} \times \mathbb{P}^{n} \rightarrow \mathbb{P}$ is contractive for the Thompson metric;

(iii) the equation

$$
X=\Lambda\left(\omega ; X \#_{t} A_{1}, \ldots, X \#_{t} A_{n}\right)
$$

has a unique solution in $\mathbb{P}$ for all $t \in(0,1), \omega \in \Delta_{n}$ and $\mathbb{A} \in \mathbb{P}^{n}$;

(iv) $\mathcal{K}(\omega ; \mathbb{A})=\{\Lambda(\omega ; \mathbb{A})\}$ for all $\omega \in \Delta_{n}$ and $\mathbb{A} \in \mathbb{P}^{n}$.

Furthermore, $X=\Lambda\left(\omega ; X \#_{t} A_{1}, \ldots, X \#_{t} A_{n}\right)$ if and only if $X=\Lambda\left(\omega ; A_{1}, \ldots, A_{n}\right)$, and $X=\Lambda\left(\omega ; A_{1}, \ldots, A_{n-1}, X\right)$ if and only if $X=\Lambda\left(\hat{\omega} ; A_{1}, \ldots, A_{n-1}\right)$ for all $t \in[0,1]$ and $\omega \in \Delta_{n}$ and $A_{i} \in \mathbb{P}, i=1, \ldots, n$. 
Proof. We first show the $\Lambda$ satisfies condition (iii). Fix $\omega \in \Delta_{n}$ and $\mathbb{A}=\left(A_{1}, \ldots, A_{n}\right)$. Define $f_{t}: \mathbb{P} \rightarrow \mathbb{P}$ defined by $f_{t}(X)=\Lambda\left(\omega ; X \#_{t} A_{1}, \ldots, X \#_{t} A_{n}\right)$. Then

$$
\begin{aligned}
d\left(f_{t}(X), f_{t}(Y)\right) & =d\left(\Lambda\left(\omega ; X \#_{t} A_{1}, \ldots, X \#_{t} A_{n}\right), \Lambda\left(\omega ; Y \#_{t} A_{1}, \ldots, Y \#_{t} A_{n}\right)\right. \\
& \leq d\left(X \#_{t} A_{j}, Y \#_{t} A_{j}\right):=\max \left\{d\left(X \#_{t} A_{i}, Y \#_{t} A_{i}\right): 1 \leq i \leq n\right\} \\
& \leq(1-t) d(X, Y)
\end{aligned}
$$

where the first inequality follows from Theorem 4.4(P5) and the second from Lemma 2.2(iii). It follows that $f_{t}$ is a strict contraction for the Thompson metric and hence has a unique fixed point, which is the unique solution for the equation of (iii).

Next, we establish the equivalence between (i)-(iv).

(i) implies (ii): By Proposition 2.5 and the monotonicity of $\Lambda$ (Theorem 4.4).

(ii) implies (iii): Let $\omega=\left(w_{1}, \ldots, w_{n}\right) \in \Delta_{n}$ and let $\mathbb{A}=\left(A_{1}, \ldots, A_{n}\right) \in \mathbb{P}^{n}$. For $t \in(0,1)$, it follows from Lemma 2.2 (iii) and the hypothesis that the map $f_{t}: \mathbb{P} \rightarrow \mathbb{P}$ defined by $f_{t}(X)=\Lambda\left(\omega ; X \#_{t} A_{1}, \ldots, X \#_{t} A_{n}\right)$ is a strict contraction for the Thompson metric and hence has a unique fixed point on $\mathbb{P}$, i.e., $X=$ $\Lambda\left(\omega ; X \#_{t} A_{1}, \ldots, X \#_{t} A_{n}\right)$ has a unique solution in $\mathbb{P}$.

(iii) implies (iv): Let $X \in \mathcal{K}\left(\omega ; A_{1}, \ldots, A_{n}\right)$ and pick $\epsilon_{\omega}>1$ as in Theorem 6.3. Pick $t \in(0,1)$ such that

$$
\left(X^{-1 / 2} A_{i} X^{-1 / 2}\right)^{t} \in\left[\epsilon_{\omega}^{-1} I, \epsilon_{\omega} I\right], \quad i=1, \ldots, n .
$$

Then $\sum_{i=1}^{n} w_{i} \log \left(X^{-1 / 2} A_{i} X^{-1 / 2}\right)=0=\sum_{i=1}^{n} w_{i} \log \left(\left(X^{-1 / 2} A_{i} X^{-1 / 2}\right)^{t}\right)$. Clearly $Y=I$ is then a solution of $\sum_{i=1}^{n} w_{i} \log \left(Y^{-1 / 2}\left(X^{-1 / 2} A_{i} X^{-1 / 2}\right)^{t} Y^{-1 / 2}\right)=0$, and by the monotonicity and idempotency of $\Lambda$,

$$
\Lambda\left(\omega ;\left(X^{-1 / 2} A_{1} X^{-1 / 2}\right)^{t}, \ldots,\left(X^{-1 / 2} A_{n} X^{-1 / 2}\right)^{t}\right)
$$

must belong to $\left[\epsilon_{\omega}^{-1} I, \epsilon_{\omega} I\right]$. By the uniqueness of the Karcher solution on $\left[\epsilon_{\omega}^{-1} I, \epsilon_{\omega} I\right]$ (Theorem 6.3),

$$
I=\Lambda\left(\omega ;\left(X^{-1 / 2} A_{1} X^{-1 / 2}\right)^{t}, \ldots,\left(X^{-1 / 2} A_{n} X^{-1 / 2}\right)^{t}\right) .
$$

By invariancy under congruence transformations (property (P6) of Theorem 4.4),

$$
\begin{aligned}
X & =\Lambda\left(\omega ; X^{1 / 2}\left(X^{-1 / 2} A_{1} X^{-1 / 2}\right)^{t} X^{1 / 2}, \ldots, X^{1 / 2}\left(X^{-1 / 2} A_{n} X^{-1 / 2}\right)^{t} X^{1 / 2}\right) \\
& =\Lambda\left(\omega ; X \#_{t} A_{1}, \ldots, X \#_{t} A_{n}\right) .
\end{aligned}
$$

Since $\Lambda\left(\omega ; A_{1}, \ldots, A_{n}\right) \in \mathcal{K}(\omega ; \mathbb{A})$ is one possibility for our original choice of $X$, property (iii) implies $X=\Lambda(\omega ; \mathbb{A})$. This shows that $\mathcal{K}(\omega ; \mathbb{A})=\{\Lambda(\omega ; \mathbb{A})\}$ and that $\Lambda(\omega ; \mathbb{A})$ is the unique solution of $X=\Lambda\left(\omega ; X \#_{t} A_{1}, \ldots, X \#_{t} A_{n}\right)$.

(iv) implies (i): Follows from Lemma 6.1(1).

Finally, suppose that one of the equivalent conditions (i)-(iv) holds true. Let $X=\Lambda\left(\hat{\omega} ; A_{1}, \ldots, A_{n-1}\right)$. Then $\sum_{i=1}^{n-1} \frac{w_{i}}{1-w_{n}} \log \left(X^{-1 / 2} A_{i} X^{-1 / 2}\right)=0$ and hence

$$
0=\sum_{i=1}^{n-1} \frac{w_{i}}{1-w_{n}} \log \left(X^{-1 / 2} A_{i} X^{-1 / 2}\right)+\frac{w_{n}}{1-w_{n}} \log \left(X^{-1 / 2} X X^{-1 / 2}\right) .
$$

By (iv), $X \in \mathcal{K}\left(\omega ; A_{1}, \ldots, A_{n-1}, X\right)=\left\{\Lambda\left(\omega ; A_{1}, \ldots, A_{n-1}, X\right)\right\}$, that is,

$$
X=\Lambda\left(\omega ; A_{1}, \ldots, A_{n-1}, X\right) .
$$


By (ii) the map $f: \mathbb{P} \rightarrow \mathbb{P}$ defined by $f(Z)=\Lambda\left(\omega ; A_{1}, \ldots, A_{n-1}, Z\right)$ is a strict contraction for the Thompson metric and has a unique fixed point. Thus

$$
\Lambda\left(\omega ; A_{1}, \ldots, A_{n-1}, Z\right)=Z
$$

if and only if $Z=X=\Lambda\left(\hat{\omega} ; A_{1}, \ldots, A_{n-1}\right)$.

We isolate as a separate theorem the main result of the preceding, a restatement of item (iv).

Theorem 6.5. For $A_{1}, \ldots, A_{n} \in \mathbb{P}$, the Karcher mean $X=\Lambda\left(\omega ; A_{1}, \ldots, A_{n}\right)$ is the unique solution of the Karcher equation

$$
0=\sum_{i=1}^{n} \omega_{i} \log \left(X^{-1 / 2} A_{i} X^{-1 / 2}\right) .
$$

Remark 6.6. In light of Theorem 6.5 it is more natural to define the Karcher mean $\Lambda\left(\omega ; A_{1}, \ldots, A_{n}\right)$ to be the unique solution of the corresponding Karcher equation. That was certainly our motivation in naming it the Karcher mean from the beginning.

By Corollary $5.7 \Lambda^{*}(\omega ; \mathbb{A})=\lim _{t \rightarrow 0^{-}} P_{t}(\omega ; \mathbb{A})$ also satisfies the same Karcher equation as $\Lambda(\omega ; \mathbb{A})$ and hence by the uniqueness of solution, the two are equal. This yields the following corollary.

Corollary 6.7. For a weight $\omega=\left(w_{1}, \ldots w_{n}\right) \in \Delta_{n}$ and $\mathbb{A}=\left(A_{1}, \ldots, A_{n}\right) \in \mathbb{P}^{n}$, $\Lambda^{*}(\omega ; \mathbb{A})=\Lambda(\omega ; \mathbb{A})$, and thus $\Lambda(\omega ; \mathbb{A})=\lim _{t \rightarrow 0} P_{t}(\omega ; \mathbb{A})$.

We gather together our results about the fundamental properties of the Karcher mean.

Theorem 6.8. For a weight $\omega=\left(w_{1}, \ldots w_{n}\right) \in \Delta_{n}$ and $\mathbb{A}=\left(A_{1}, \ldots, A_{n}\right) \in \mathbb{P}^{n}$, the following properties hold.

(P1) (Consistency with scalars) $\Lambda(\omega ; \mathbb{A})=A_{1}^{w_{1}} \cdots A_{n}^{w_{n}}$ if the $A_{i}$ 's commute;

(P2) (Joint homogeneity) $\Lambda\left(\omega ; a_{1} A_{1}, \ldots, a_{n} A_{n}\right)=a_{1}^{w_{1}} \cdots a_{n}^{w_{n}} \Lambda(\omega ; \mathbb{A})$;

(P3) (Permutation invariance)

$$
\Lambda\left(\omega_{\sigma} ; \mathbb{A}_{\sigma}\right)=\Lambda(\omega ; \mathbb{A}) \text {, where } \omega_{\sigma}=\left(w_{\sigma(1)}, \ldots, w_{\sigma(n)}\right) ;
$$

(P4) (Monotonicity) if $B_{i} \leq A_{i}$ for all $1 \leq i \leq n$, then $\Lambda(\omega ; \mathbb{B}) \leq \Lambda(\omega ; \mathbb{A})$;

(P5) (Continuity) $\Lambda$ is contractive for the Thompson metric;

(P6) (Congruence invariance) $\Lambda\left(\omega ; M^{*} \mathbb{A} M\right)=M^{*} \Lambda(\omega ; \mathbb{A}) M$ for any invertible $M$;

(P7) (Joint concavity) $\Lambda(\omega ; \lambda \mathbb{A}+(1-\lambda) \mathbb{B}) \geq \lambda \Lambda(\omega ; \mathbb{A})+(1-\lambda) \Lambda(\omega ; \mathbb{B})$ for $0 \leq \lambda \leq 1$

(P8) Self-duality) $\Lambda\left(\omega ; A_{1}^{-1}, \ldots, A_{n}^{-1}\right)^{-1}=\Lambda\left(\omega ; A_{1}, \ldots, A_{n}\right)$; and

(P9) (AGH weighted mean inequalities)

$$
\left(\sum_{i=1}^{n} w_{i} A_{i}^{-1}\right)^{-1} \leq \Lambda(\omega ; \mathbb{A}) \leq \sum_{i=1}^{n} w_{i} A_{i} .
$$

Proof. By Theorem 4.4 and Theorem 6.4. $\Lambda$ is jointly homogeneous and is contractive for the Thompson metric. The remaining properties appear in Theorem 4.4. except that $(\mathrm{P} 8)$ is modified in light of the preceding corollary. 
Remark 6.9. The Karcher mean is uniquely determined by congruence invariancy $(P 6)$, self-duality $(P 8)$, and the following property

$$
\sum_{i=1}^{n} w_{i} \log A_{i} \leq 0 \quad \text { implies } \quad \Lambda\left(\omega ; A_{1}, \ldots, A_{n}\right) \leq I
$$

for all $\omega \in \Delta_{n}$ and $\left(A_{1}, \ldots, A_{n}\right) \in \mathbb{P}^{n}$. Furthermore, $\Lambda\left(\omega ; A_{1}, \ldots, A_{n}\right) \leq I$ implies that $\Lambda\left(\omega ; A_{1}^{p}, \ldots, A_{n}^{p}\right) \leq I$ for all $p \geq 1$, and the following statements are equivalent;

(i) $\sum_{i=1}^{n} \log A_{i} \leq 0$;

(ii) $\Lambda\left(\frac{1}{n}, \ldots, \frac{1}{n} ; \bar{A}_{1}^{p}, \ldots, A_{n}^{p}\right) \leq I$ for all $p>0$; and

(iii) $\Lambda\left(\omega_{\mathbf{p}} ; A_{1}^{p_{1}}, \ldots, A_{n}^{p_{n}}\right) \leq I$ for all $\mathbf{p}=\left(p_{1}, \ldots, p_{n}\right) \in \mathbb{R}_{+}^{n}$, where

$$
\omega_{\mathbf{p}}=\frac{1}{\sum_{i=1}^{n} \mathbf{p}_{\neq i}}\left(\mathbf{p}_{\neq 1}, \ldots, \mathbf{p}_{\neq n}\right) \in \Delta_{n}, \quad \mathbf{p}_{\neq i}:=\prod_{j \neq i} p_{j} .
$$

For a finite-dimensional Hilbert space, property $(Y)$ and the previous characterization for the Karcher mean appear in 24 and 17, respectively. The proofs are heavily dependent on property (Y) and Hansen's inequality and are similar to those of $[17,24$.

\section{SUbAlGEBRAS}

For convenience and ease of presentation we have limited our considerations to the full algebra $\mathcal{B}(H)$ of bounded linear operators. However, we observe that the constructions can be carried out in large classes of subalgebras (which we assume always to contain the identity $I$ ). For any norm-closed $C^{*}$-subalgebra $\mathcal{A}$ of $\mathcal{B}(H)$, $\mathbb{P}_{\mathcal{A}}=\mathcal{A} \cap \mathbb{P}$ will be its open cone of positive operators, and will be closed under the operation of taking weighted geometric means $A \#_{t} B$. Hence it will be closed under taking power means $P_{t}(\omega ; \mathbb{A})$, since the power mean is the limit in the Thompson metric of a contractive map defined from the weighted geometric means on $\mathbb{P}_{\mathcal{A}}$, and since the topology of the Thompson metric agrees with the relative operator norm topology. Since we have defined $\Lambda(\omega ; \mathbb{A})$ to be the strong limit of the monotonically decreasing family $\left\{P_{t}: t>0\right\}$, we need that the subalgebra is monotone complete (actually, monotone $\sigma$-complete will suffice since one can restrict to $t=1 / n$ and obtain the same infimum). Once one has closure under the Karcher mean for the subalgebra, then one sees readily that its properties that we have derived for the full algebra $\mathcal{B}(H)$ are inherited by the subalgebra, in particular its characterization as the unique solution of the corresponding Karcher equation. Since the von Neumann subalgebras are strongly closed, they in particular have Karcher means defined in the manner of this paper and satisfying the properties derived for it.

\section{Open PROBlems}

We close with three open problems:

Problem 1: Is the strong convergence $\lim _{t \rightarrow 0} P_{t}=\Lambda$ of the power means to the Karcher mean actually convergence in the operator norm topology?

Problem 2: For a given weight $\omega$, is the map $\left(A_{1}, \ldots, A_{n}\right) \mapsto \Lambda\left(\omega ; A_{1}, \ldots, A_{n}\right)$ from $\mathbb{P}^{n}$ to $\mathbb{P}$ a $C^{\infty}$-map? (We established this in a neighborhood of the diagonal.)

Problem 3: For a given weight $\omega$, is the map $\left(A_{1}, \ldots, A_{n}\right) \mapsto \Lambda\left(\omega ; A_{1}, \ldots, A_{n}\right)$ from $\mathbb{P}^{n}$ to $\mathbb{P}$ strongly continuous on order intervals $\left[e^{-m} I, e^{m} I\right]$ ? 


\section{ACKNOWLEDGEMENT}

The authors wish to express their gratitude to Professor Dick Kadison for helpful insights about the strong topology, especially for his pointing us to reference [9]. The authors also thank the referee for helpful comments, particularly for pointing out a needed correction in the authors' use of the Implicit Function Theorem in the proof of Theorem 6.3.

\section{REFERENCES}

[1] T. Ando, Chi-Kwong Li, and Roy Mathias, Geometric means, Linear Algebra Appl. 385 (2004), 305-334, DOI 10.1016/j.laa.2003.11.019. MR2063358(2005f:47049)

[2] Marcel Berger, A panoramic view of Riemannian geometry, Springer-Verlag, Berlin, 2003. MR2002701 (2004h:53001)

[3] Rajendra Bhatia, On the exponential metric increasing property, Linear Algebra Appl. 375 (2003), 211-220, DOI 10.1016/S0024-3795(03)00647-5. MR2013466 (2004m:53032)

[4] Rajendra Bhatia, Positive definite matrices, Princeton Series in Applied Mathematics, Princeton University Press, Princeton, NJ, 2007. MR2284176 (2007k:15005)

[5] Rajendra Bhatia and John Holbrook, Riemannian geometry and matrix geometric means, Linear Algebra Appl. 413 (2006), no. 2-3, 594-618, DOI 10.1016/j.laa.2005.08.025. MR2198952 (2007c:15030)

[6] Martin R. Bridson and André Haefliger, Metric spaces of non-positive curvature, Grundlehren der Mathematischen Wissenschaften [Fundamental Principles of Mathematical Sciences], vol. 319, Springer-Verlag, Berlin, 1999. MR1744486 (2000k:53038)

[7] G. Corach, H. Porta, and L. Recht, Convexity of the geodesic distance on spaces of positive operators, Illinois J. Math. 38 (1994), no. 1, 87-94. MR.1245836 (94i:58010)

[8] Karl E. Gustafson and Duggirala K. M. Rao, Numerical range, Universitext, Springer-Verlag, New York, 1997. The field of values of linear operators and matrices. MR 1417493(98b:47008)

[9] Richard V. Kadison, Strong continuity of operator functions, Pacific J. Math. 26 (1968), 121-129. MR0231211 (37 \#6766)

[10] H. Karcher, Riemannian center of mass and mollifier smoothing, Comm. Pure Appl. Math. 30 (1977), no. 5, 509-541. MR0442975 (56 \#1350)

[11] Fumio Kubo and Tsuyoshi Ando, Means of positive linear operators, Math. Ann. 246 (1979/80), no. 3, 205-224, DOI 10.1007/BF01371042. MR563399 (84d:47028)

[12] Serge Lang, Fundamentals of differential geometry, Graduate Texts in Mathematics, vol. 191, Springer-Verlag, New York, 1999. MR1666820 (99m:53001)

[13] Jimmie D. Lawson and Yongdo Lim, The geometric mean, matrices, metrics, and more, Amer. Math. Monthly 108 (2001), no. 9, 797-812, DOI 10.2307/2695553. MR1864051 (2002h:15023)

[14] Jimmie Lawson and Yongdo Lim, Metric convexity of symmetric cones, Osaka J. Math. 44 (2007), no. 4, 795-816. MR2383810 (2009f:46095)

[15] Jimmie Lawson and Yongdo Lim, A general framework for extending means to higher orders, Colloq. Math. 113 (2008), no. 2, 191-221, DOI 10.4064/cm113-2-3. MR2425082 (2009f:47028)

[16] Jimmie Lawson and Yongdo Lim, Monotonic properties of the least squares mean, Math. Ann. 351 (2011), no. 2, 267-279, DOI 10.1007/s00208-010-0603-6. MR2836658

[17] Yongdo Lim and Miklós Pálfia, Matrix power means and the Karcher mean, J. Funct. Anal. 262 (2012), no. 4, 1498-1514, DOI 10.1016/j.jfa.2011.11.012. MR2873848(2012m:15025)

[18] Maher Moakher, A differential geometric approach to the geometric mean of symmetric positive-definite matrices, SIAM J. Matrix Anal. Appl. 26 (2005), no. 3, 735-747 (electronic), DOI 10.1137/S0895479803436937. MR2137480 (2005m:15059)

[19] Roger D. Nussbaum and Joel E. Cohen, The arithmetic-geometric mean and its generalizations for noncommuting linear operators, Ann. Scuola Norm. Sup. Pisa Cl. Sci. (4) 15 (1988), no. 2, 239-308 (1989). MR1007399 (90e:47018)

[20] Roger D. Nussbaum, Finsler structures for the part metric and Hilbert's projective metric and applications to ordinary differential equations, Differential Integral Equations 7 (1994), no. 5-6, 1649-1707. MR 1269677 (95b:58010) 
[21] Karl-Theodor Sturm, Probability measures on metric spaces of nonpositive curvature, (Paris, 2002), Contemp. Math., vol. 338, Amer. Math. Soc., Providence, RI, 2003, pp. 357-390, DOI 10.1090/conm/338/06080. MR2039961 (2005d:60009)

[22] A. C. Thompson, On certain contraction mappings in a partially ordered vector space., Proc. Amer. Math. soc. 14 (1963), 438-443. MR0149237 (26 \#6727)

[23] Joachim Weidmann, Linear operators in Hilbert spaces, Graduate Texts in Mathematics, vol. 68, Springer-Verlag, New York, 1980. Translated from the German by Joseph Szücs. MR.566954 (81e:47001)

[24] T. Yamazaki, The Riemannian mean and matrix inequalities related to the Ando-Hiai inequality and chaotic order, Operators and Matrices 6 (2012), 577-588.

Department of Mathematics, Louisiana State University, Baton Rouge, Louisiana 70803

E-mail address: lawson@math.lsu.edu

Department of Mathematics, Sungkyunkwan University, Suwon 440-746, Korea

E-mail address: ylim@skku.edu 